Preprint typeset in JHEP style - HYPER VERSION

hep-th/0301150

AEI-2003-007

\title{
Conformal Dimensions of Two-Derivative BMN Operators
}

\author{
Thomas Klose \\ Max-Planck-Institut für Gravitationsphysik \\ Albert-Einstein-Institut \\ Am Mühlenberg 1, D-14476 Golm, Germany \\ E-mail: thomas.klose@aei.mpg.de
}

\begin{abstract}
We compute the anomalous dimensions of BMN operators with two covariant derivative impurities at the planar level up to first order in the effective coupling $\lambda^{\prime}$. The result equals those for two scalar impurities as well as for mixed scalar and vector impurities given in the literature. Though the results are the same, the computation is very different from the scalar case. This is basically due to the existence of a non-vanishing overlap between the derivative impurity and the "background" field $Z$. We present details of these differences and their consequences.
\end{abstract}

Keywords: AdS-CFT Correspondence, Penrose limit and pp-wave background, 1/N Expansion. 


\section{Contents}

1. Introduction

2. Two-derivative BMN operators and their conformal dimensions

3. The one-loop computation 6

3.1 Preliminary remarks 6

3.2 Classical Correlator $\mathcal{O}\left(g_{\text {YM }}^{0}\right)$

3.3 Quantum Corrections $\mathcal{O}\left(g_{\text {YM }}^{2}\right)$

3.3.1 Vertex without any derivative 16

3.3.2 Vertex with one derivative 16

3.3.3 Vertex with two derivatives 17

3.3.4 Vertex with three derivatives 19

3.3.5 Vertex with four derivatives 20

3.3 .6 Summary 21

3.4 Anomalous dimension 23

4. Conclusion 23

A. Appendix: Yang-Mills theory 25

\section{Introduction}

The Berenstein-Maldacena-Nastase (BMN) correspondence [1] is a limit of the AdS/CFT duality [2] between type IIB superstring theory on $A d S_{5} \times S^{5}$ and $\mathcal{N}=4$ supersymmetric Yang-Mills theory on $\mathbb{R}^{4}$. The novel feature is that there exists a regime of the effective coupling strengths where both theories can be treated perturbatively at the same time. On the string theory side the BMN limit amounts to a Penrose limit [3] of $A d S_{5} \times S^{5}$ and leads to a plane-wave geometry [4] which is labeled by a parameter $\mu$ of unit mass dimension. It is the geometry which a particle experiences that travels with large angular momentum $J$ along a circle $S^{1}$ on the 5 -sphere $S^{5}$. Choosing one particular circle corresponds in the super Yang-Mills theory to singling out a U(1) subgroup of the SU(4) $R$-symmetry group. In consequence, the string states are represented by operators with large $U(1)_{R}$ charge $J$ which are commonly named BMN operators.

The correspondence is made more precise by the following dictionary. The string theory Hamiltonian $\hat{H}^{\text {l.c. }}$ in light-cone gauge is identified with the Yang-Mills dilatation operator $\hat{D}$ and the generator $\hat{J}$ of $\mathrm{U}(1)_{R}$ transformations by the relation

$$
\frac{1}{\mu} \hat{H}^{1 . c .} \stackrel{!}{=} \hat{D}-\hat{J}
$$


In other words, this is an identification of the string energy with the conformal dimension minus the $R$-charge of the BMN operator. The parameters of both theories are related in the following way

$$
\lambda^{\prime}:=\frac{g_{\mathrm{YM}}^{2} N}{J^{2}}=\frac{1}{\left(\mu p^{+} \alpha^{\prime}\right)^{2}} \quad, \quad g_{2}:=\frac{J^{2}}{N}=4 \pi g_{s}\left(\mu p^{+} \alpha^{\prime}\right)^{2},
$$

where $g_{\mathrm{YM}}$ is the Yang-Mills coupling constant, $N$ is the rank of the gauge group $\mathrm{U}(N), g_{s}$ is string coupling constant and $p^{+}$the string light-cone momentum. In the BMN limit one takes

$$
N, J \rightarrow \infty \text { such that } \lambda^{\prime}, g_{2} \text { fixed . }
$$

Hence $\lambda^{\prime}$ and $g_{2}$ are the relevant parameters in the BMN limit. They control the quantum loop and the genus expansion, respectively. As opposed to the 't Hooft limit [5] $(N \rightarrow \infty$ with $\lambda:=g_{\mathrm{YM}}^{2} N=$ fixed) it has been found [6] [7] that in the BMN limit also non-planar diagrams contribute on the gauge theory side. At each order in $\lambda^{\prime}$ there is a series of diagrams with increasing genera controlled by $g_{2}$. In order to simplify computations one could concentrate on planar diagrams by setting $g_{2}=0$. On the string theory side the two expansions have the following significance. The light-cone string in the plane-wave background is in fact a massive string and its mass is given by $\mu \sim 1 / \sqrt{\lambda^{\prime}}$. The other parameter $g_{2} \sim g_{s}$ determines the strength of string splitting and joining.

The relationship (1.1) has been written as an operator equation. However, both sides act on very different Hilbert spaces. Therefore it is necessary to know how string states are translated into BMN operators and vice versa. This question is a subject of current research [8]-12]. Since the original proposal of BMN a lot of work has been done to understand the correspondence in more detail especially at the interacting level. Some further references for investigations on the gauge theory side are [13]- 224] and for the string theory side see e. g. [25]-41]. In [42] [43] 44] a string bit formalism has been developed which interpolates between the two sides.

In the following we summarize the mapping between string states and BMN operators at the planar level $\left(g_{2}=0\right)$ where the dictionary is well established. In this case the Hamiltonian simplifies to that of a non-interacting massive string which can be solved exactly [25] 26]. In terms of occupation numbers $N_{k}$ the Hamiltonian reads

$$
\frac{1}{\mu} \hat{H}^{\text {l.c. }}=\sum_{k=-\infty}^{\infty} N_{k} \sqrt{1+\lambda^{\prime} k^{2}},
$$

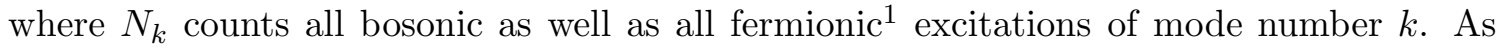
opposed to the ordinary case, for the massive string there is a pair of (bosonic) creation and annihilation operators $a_{k}^{\dagger i}, a_{k}^{i}$ independent for all modes $-\infty<k<\infty$. The index $i$ runs from 1 to 8 corresponding to the number of independent oscillators in light-cone gauge.

\footnotetext{
${ }^{1}$ In the following we will mainly concentrate on the bosonic part of the theory.
} 
In the diagonal basis underlying (1.4) the relation (1.1) becomes

$$
\sum_{k=-\infty}^{\infty} N_{k} \sqrt{1+\lambda^{\prime} k^{2}}=\Delta-J
$$

where $\Delta=\Delta^{(0)}+\delta \Delta$ is the conformal dimension of the corresponding operator. It is given by the engineering conformal dimension $\Delta^{(0)}$ corrected by the anomalous dimension $\delta \Delta$ which is due to quantum effects.

The (single) string vacuum $\left|0, p^{+}\right\rangle$has zero energy and therefore should be represented by an operator whose conformal dimension equals its $R$-charge. The operator with this property, proposed by BMN [1], is

$$
\mathcal{O}(x) \sim \operatorname{tr} Z^{J}(x)
$$

where $Z$ is that combination of Yang-Mills scalar fields with unit $R$-charge, cf. appendix A. This operator $\mathcal{O}$ is protected, i. e. its engineering conformal dimension does not receive quantum corrections. Table 1 summarizes the engineering conformal dimensions and $R$ charges of the fields of the Yang-Mills theory. For composite operators these values add up.

The excited states

$$
a_{k_{1}}^{\dagger i_{1}} \cdots a_{k_{l}}^{\dagger i_{l}}\left|0, p^{+}\right\rangle
$$

are divided into supergravity states where only zero mode oscillators are

\begin{tabular}{l|c|c|c|c|c|c} 
& $Z$ & $\bar{Z}$ & $\phi_{i}$ & $D_{\mu}$ & $\psi_{\alpha}$ & $\bar{\psi}_{\alpha}$ \\
\hline$\Delta^{(0)}$ & 1 & 1 & 1 & 1 & $3 / 2$ & $3 / 2$ \\
$J$ & 1 & -1 & 0 & 0 & $1 / 2$ & $-1 / 2$
\end{tabular}

Table 1: Engineering conformal dimensions $\Delta^{(0)}$ and $R$-charges $J$ applied $\left(k_{1}=\ldots=k_{l}=0\right)$ and non-supergravity states where the level matching condition demands $k_{1}+\ldots+k_{l}=0$. BMN operators corresponding to excited states are derived from (1.6) by inserting so-called "impurities" in-between the $Z$ 's. Excitations along the directions $i=5,6,7,8$ (originally on $S^{5}$ ) are generated by scalar field impurities $\phi_{i-4}$ whereas the insertion of covariant derivatives $D_{\mu}$ models excitations along the remaining directions $\mu=1,2,3,4$ (originally on $A d S_{5}$ ). Fermionic excitations are obtained by inserting gaugino fields $\psi_{\alpha}(\alpha=1, \ldots, 8)$. For higher excitations where there is more than one impurity one sums over all possible insertion points. If one performs this sum with appropriate phase factors which encode the mode numbers, one can realize non-supergravity modes. Some examples are given in table 2 .

Eq. (1.5) has been verified for operators with scalar field impurities to second order in $\lambda^{\prime}$ in [13] and to all orders in [17], and for mixed scalar field and covariant derivative impurities up to $\mathcal{O}\left(\lambda^{\prime}\right)$ in [19]. In this paper we confirm 1.5) to first order in $\lambda^{\prime}$ for operators with two covariant derivative impurities. On the string theory side (at least at the non-interacting level) there is no difference between the oscillation modes in the directions $\mu=1,2,3,4$ and $i=5,6,7,8$. However the gauge theory calculations with derivative impurities are very different from the case of scalar impurities, in fact they are much more complex. This is ultimately due to the overlap between derivative impurity and "background" field $Z$ which vanishes for scalar impurities. Furthermore one has to cope 


\begin{tabular}{llll} 
String state & BMN operator & occupation no & (bare) energy \\
\hline$\left|0, p^{+}\right\rangle$ & $\operatorname{tr} Z^{J}$ & $N_{k}=0$ & $\Delta-J=0$ \\
$a_{0}^{\dagger i}\left|0, p^{+}\right\rangle$ & $\operatorname{tr} \phi_{i-4} Z^{J}, \quad(i=5, \ldots, 8)$ & $N_{k}=\delta_{k 0}$ & $\Delta-J=1$ \\
$a_{0}^{\dagger \mu}\left|0, p^{+}\right\rangle$ & $\operatorname{tr}\left(D_{\mu} Z\right) Z^{J-1}, \quad(\mu=1, \ldots, 4)$ & $N_{k}=\delta_{k 0}$ & $\Delta-J=1$ \\
$a_{0}^{\dagger i} a_{0}^{\dagger \mu}\left|0, p^{+}\right\rangle$ & $\sum_{p=0}^{J-1} \operatorname{tr} \phi_{i-4} Z^{p}\left(D_{\mu} Z\right) Z^{J-p-1}$ & $N_{k}=2 \delta_{k 0}$ & $\Delta-J=2$ \\
& $\quad+\operatorname{tr}\left(D_{\mu} \phi_{i-4}\right) Z^{J}$ & & \\
$a_{n}^{\dagger 5} a_{-n}^{\dagger 6}\left|0, p^{+}\right\rangle$ & $\sum_{p=0}^{J} \operatorname{tr} \phi_{1} Z^{p} \phi_{2} Z^{J-p} e^{2 \pi i n p / J}$ & $N_{k}=\delta_{k n}+\delta_{k,-n}$ & $\Delta^{(0)}-J=2$
\end{tabular}

Table 2: Examples of string operator correspondence. All states have light-cone momentum $p^{+}$ corresponding to $R$-charge $J$.

with more diagrams stemming from insertions of the gauge field that is contained in the derivative impurity. Therefore it is very interesting to see how the result emerges in this more intricate case.

The paper is organized as follows. In the next section we introduce the operators we want to study and discuss some of their properties. In section 3 we present in detail the computation of their one-loop anomalous dimensions in the planar BMN limit. We conclude by summarizing the specialties of this computation in section 4 . Our notation and conventions can be found in the appendix.

\section{Two-derivative BMN operators and their conformal dimensions}

In this work we are concerned with operators with two covariant derivative insertions $D_{\mu}$ and $D_{\nu}$. These represent the string states $\left(\alpha_{n}^{\mu}\right)^{\dagger}\left(\alpha_{-n}^{\nu}\right)^{\dagger}\left|0, p^{+}\right\rangle$with energy

$$
\frac{1}{\mu} H=2 \sqrt{1+\lambda^{\prime} n^{2}}=2+\lambda^{\prime} n^{2}+\mathcal{O}\left(\lambda^{\prime 2}\right) .
$$

In analogy to the case of scalar field insertions we define (for $n \neq 0$ )

$$
\mathcal{D}_{\mu \nu, n}:=\frac{J^{-1 / 2}}{2 N_{0}^{J}}\left[\sum_{p=1}^{J-1} \operatorname{tr}\left(D_{\mu} Z\right) Z^{p-1}\left(D_{\nu} Z\right) Z^{J-1-p} e^{2 \pi i n p / J}+\operatorname{tr}\left(D_{\mu} D_{\nu} Z\right) Z^{J-1}\right],
$$

where all fields are located at point $x$ and $N_{0}:=\sqrt{g_{\mathrm{YM}}^{2} N / 8 \pi^{2}}$. These operators carry $R$-charge $J$ and possess engineering conformal dimension

$$
\Delta^{(0)}=J+2 .
$$

We note that the covariant derivatives in the second term commute because of the trace. In particular the term

$$
\operatorname{tr}\left[\partial_{\mu} A_{\nu}, Z\right] Z^{J-1}=\operatorname{tr} \partial_{\mu} A_{\nu}\left[Z, Z^{J-1}\right]=0
$$

vanishes which will become important later since it implies that we may choose whether we want to differentiate the gauge field or rather not. As a consequence of this commutation we have the identity

$$
\mathcal{D}_{\mu \nu,-n}=\mathcal{D}_{\nu \mu, n}
$$


and therefore we could restrict ourselves to operators with positive index $n>0$.

For the definition of the zero mode operators $(n=0)$ we take $(2.2)$ with an additional normalization factor of $J^{-1}$. This is natural since $\sum e^{2 \pi i n p / J}$ is of order $\mathcal{O}(J)$ for $n=0$ and of order $\mathcal{O}(1)$ otherwise. Apart from that, the definition (2.2) simplifies drastically in absence of the phase factor. The commutator terms cancel and the derivatives can be taken out of the trace:

$$
\begin{aligned}
\mathcal{D}_{\mu \nu, 0} & :=\frac{J^{-3 / 2}}{2 N_{0}^{J}}\left[\sum_{p=1}^{J-1} \operatorname{tr}\left(D_{\mu} Z\right) Z^{p-1}\left(D_{\nu} Z\right) Z^{J-1-p}+\operatorname{tr}\left(D_{\mu} D_{\nu} Z\right) Z^{J-1}\right] \\
& =\frac{J^{-5 / 2}}{2 N_{0}^{J}} \partial_{\mu} \partial_{\nu} \operatorname{tr} Z^{J} .
\end{aligned}
$$

This means that $\mathcal{D}_{\mu \nu, 0}$ is a descendant of the protected vacuum operator (1.6).

The set of operators (2.2), 2.6) transforms under a reducible tensor representation of $S O(4)$. A decomposition into subsets that transform under (different) irreducible representations lead to the following linear combinations $(n \in \mathbb{Z})$

$$
\begin{aligned}
\mathcal{D}_{(\mu \nu), n} & :=\frac{1}{2}\left(\mathcal{D}_{\mu \nu, n}+\mathcal{D}_{\nu \mu, n}\right)-\frac{1}{4} \delta_{\mu \nu} \mathcal{D}_{\kappa \kappa, n}, \\
\mathcal{D}_{[\mu \nu], n} & :=\frac{1}{2}\left(\mathcal{D}_{\mu \nu, n}-\mathcal{D}_{\nu \mu, n}\right) \\
\mathcal{D}_{n} & :=\mathcal{D}_{\kappa \kappa, n}
\end{aligned}
$$

which represent the symmetric-traceless part, the anti-symmetric part and the trace, respectively.

Our aim now is to compute the conformal dimensions $\Delta_{n}=\Delta^{(0)}+\delta \Delta_{n}$ of these operators. The notation anticipates that the conformal dimensions will depend on the mode number but not on the $S O(4)$ irrep. They can be read off from the two point correlator whose generic form is

$$
\begin{aligned}
\left\langle\mathcal{D}_{\mu \nu, n}(x) \overline{\mathcal{D}}_{\rho \sigma, m}(0)\right\rangle & =\frac{C_{\mu \nu, \rho \sigma}(x) \delta_{n, m}+C_{\mu \nu, \sigma \rho}(x) \delta_{n,-m}}{\left(x^{2}\right)^{\Delta_{n}}} \\
& \approx \frac{C_{\mu \nu, \rho \sigma}(x) \delta_{n, m}+C_{\mu \nu, \sigma \rho}(x) \delta_{n,-m}}{\left(x^{2}\right)^{\Delta^{(0)}}}\left(1+\delta \Delta_{n} \ln x^{-2}\right) .
\end{aligned}
$$

Conformal invariance dictates more specifically the form of the function $C_{\mu \nu, \rho \sigma}(x)$. It is built out of Kronecker deltas $\delta_{\mu \nu}$, which connect indices referring to the same tangent space, and inversion matrices $J_{\mu \rho} \equiv \delta_{\mu \rho}-\frac{2 x_{\mu} x_{\rho}}{x^{2}}$, which mediate between different tangent spaces. The particular structure in the indices $n$ and $m$ means nothing but orthogonality. The term $\delta_{n,-m}$ might appear unexpected at first sight, but it is required because of the symmetry (2.5). When restricted to $n, m \geq 0$ only the usual delta-symbol is present.

Of course it has to be checked that the correlators of the operators defined in (2.2) and (2.6) indeed have the required form (2.8). In fact, we will find that for the introduced operators this is not the case and we are compelled to redefine the non-zero mode operators (2.2) by adding a suitable proportion of the zero mode operators (2.6), cf. (3.14).

At the technical level the one-loop anomalous dimension is determined as follows. Computing the two point correlator (2.8) at tree level fixes the overall constant $C_{\mu \nu, \rho \sigma}$. 
Afterwards one obtains the relative factor, which essentially is the anomalous dimension, from the one-loop computation. In the latter calculation we use dimensional reduction to $d$ dimensions in order to regularize divergences and find the renormalized operators.

In what follows we will perform this calculation. We consider the BMN limit (1.3) and furthermore restrict ourselves to diagrams that can be drawn on a sphere without crossing lines. As mentioned in the introduction, our result

$$
\Delta_{n}=J+2+\lambda^{\prime} n^{2}
$$

equals the one for operators with scalar insertions and mixed scalar-vector insertions. Due to supersymmetry arguments 22] the matching of the conformal dimensions for operators with derivative insertions is actually expected. Nevertheless our computation provides another consistency check for the BMN proposal and we will show some specialties of our computation with regard to the scalar case.

\section{The one-loop computation}

\subsection{Preliminary remarks}

The first thing to note is that the insertion of a covariant derivative actually produces two terms, one with partial derivative and one with the commutator of the gauge field $A$. To be more concrete let us split the operator (2.2) into pieces with no, one and two $A$-fields, respectively, as

$$
\mathcal{D}_{\mu \nu, n}=d_{\mu \nu, n}^{(0)}+d_{\mu \nu, n}^{(1)}+d_{\mu \nu, n}^{(2)} .
$$

With this decomposition the two point correlator consists of 9 terms. But due to the different number of fields, it is clear that at tree level the different parts of (3.1) do not have any overlap. Moreover, since each propagator carries a factor of $g_{\mathrm{YM}}^{2}$, all three parts contribute to different orders in the coupling constant. Taking into account the normalization $N_{0}$, which also contains factors of $g_{\mathrm{YM}}$, this can be summarized by

$$
\left\langle d_{\mu \nu, n}^{(k)} \bar{d}_{\rho \sigma, m}^{(l)}\right\rangle_{\text {tree }} \sim\left(g_{\mathrm{YM}}^{2}\right)^{k} \delta_{k l} .
$$

Thus, for the zeroth order we only need to determine the tree level correlator of $\mathcal{D}_{\mu \nu, n}$ where the covariant derivatives are replaced by partial ones. Therefore define

$$
\left\langle\mathcal{D}_{\mu \nu, n} \overline{\mathcal{D}}_{\rho \sigma, m}\right\rangle_{0}:=\left\langle d_{\mu \nu, n}^{(0)} \bar{d}_{\rho \sigma, m}^{(0)}\right\rangle_{\text {tree }} .
$$

The second term in (3.1) contributes to the same order as the one-loop corrections and therefore will be included below. The third term can be disregarded because its contribution to the perturbation expansion lies beyond our objective.

In order to figure out what happens at one-loop order, we need to discuss the possible vertices. First of all there are the same interactions as in the scalar case, namely the scalar self-energy, the four scalar interaction and the gluon exchange. All of these contribute to the next-to-leading order in $g_{\mathrm{YM}}$ when inserted into the two point function of $d^{(0)}$ with itself. However, in our case there is also an additional contribution to this order stemming 
from the cross term of $d^{(0)}$ with $d^{(1)}$ which can be connected by the gluon emission vertex. Let us collect all relevant terms in the definition

$$
\begin{aligned}
\left\langle\mathcal{D}_{\mu \nu, n} \overline{\mathcal{D}}_{\rho \sigma, m}\right\rangle_{1}:= & \left\langle d_{\mu \nu, n}^{(0)} \bar{d}_{\rho \sigma, m}^{(0)}\right\rangle_{g_{\mathrm{YM}}^{2}} \\
& +\left\langle d_{\mu \nu, n}^{(1)} \bar{d}_{\rho \sigma, m}^{(0)}\right\rangle_{g_{\mathrm{YM}}}+\left\langle d_{\mu \nu, n}^{(0)} \bar{d}_{\rho \sigma, m}^{(1)}\right\rangle_{g_{\mathrm{YM}}} \\
& +\left\langle d_{\mu \nu, n}^{(1)} \bar{d}_{\rho \sigma, m}^{(1)}\right\rangle_{\mathrm{tree}},
\end{aligned}
$$

where the subscripts $g_{\mathrm{YM}}^{2}$ and $g_{\mathrm{YM}}$ refer to the order of the inserted interactions.

In the next subsections we will work out (3.3) and (3.4) in detail. For that purpose let us introduce some technicalities. For the zero mode operators the derivatives could be pulled out of the trace, cf. (2.6). But also the non-zero mode operators (2.2) can be written with the derivatives taken in front by utilizing the idea of the $q$-derivative 19.

$$
\mathcal{D}_{\mu \nu, n}=\left.\frac{J^{-3 / 2}}{2 N_{0}^{J}} \sum_{i, j=1}^{J} q^{j-i} D_{\mu}^{x_{i}} D_{\nu}^{x_{j}} \operatorname{tr} Z\left(x_{1}\right) Z\left(x_{2}\right) \cdots Z\left(x_{J}\right)\right|_{x_{1}=x_{2}=\ldots=x}
$$

where $q=e^{2 \pi i n / J}$. Here $D_{\mu}^{x_{i}}$ acts only on $Z\left(x_{i}\right)$. This notation is actually nothing but the explicit version of the $q$-derivative but it is preferred in order to easier keep track which phase factor belongs to which derivative. However, it enforced us to place all fields $Z$ at different locations $x_{1}, \ldots, x_{J}$, which are taken to coincide after the derivatives are performed. This is indicated by the vertical bar. We have not yet specified where we want the gauge field that is contained in the covariant derivative to be located at. There are basically two possibilities: either at $x_{i}$ where it will be hit by other derivatives or at $x$ where it is protected from them. As we have argued in (2.4) both choices yield the same result. In fact, we may more generally define

$$
D_{\mu}^{x_{i}}=\partial_{\mu}^{x_{i}}-i\left[a A_{\mu}\left(x_{i}\right)+b A_{\mu}(x), \quad\right]^{x_{i}}
$$

with arbitrary $a$ and $b$ as long as $a+b=1$. For our computation we choose $a=1$ and $b=0$ such that the gauge field $i s$ differentiated. And we demand even more. The usual understanding is that operators only act to the right. But in order to obtain more homogeneous expressions we would like to have, in a product of covariant derivatives, all gauge fields differentiated:

$$
D_{\mu}^{x_{i}} D_{\nu}^{x_{j}} \equiv \partial_{\mu}^{x_{i}} \partial_{\nu}^{x_{j}}-i \partial_{\mu}^{x_{i}}\left[A_{\nu}\left(x_{j}\right),\right]^{x_{j}}-i \partial_{\nu}^{x_{j}}\left[A_{\mu}\left(x_{i}\right),\right]^{x_{i}}-\left[A_{\mu}\left(x_{i}\right),\right]^{x_{i}}\left[A_{\mu}\left(x_{j}\right),\right]^{x_{j}} .
$$

If one wishes this could be called a kind of normal ordering.

There is another important property of (3.5). Although the fields $Z$ have been moved to different coordinates the cyclicity of the expression is retained. Since $q^{J}=1$ the coordinates can be relabeled cyclicly without changing the phase factor. Explicitly we have for some arbitrary $^{2}$ function $f\left(x_{1}, \ldots, x_{J}\right)$

$$
\left.\sum_{i, j=1}^{J} q^{j-i} D_{\mu}^{x_{i}} D_{\nu}^{x_{j}} f\left(x_{1}, \ldots, x_{J}\right)\right|_{x_{1}=\ldots=x}=\left.\sum_{i, j=1}^{J} q^{j-i} D_{\mu}^{x_{i}} D_{\nu}^{x_{j}} f\left(x_{2}, \ldots, x_{J}, x_{1}\right)\right|_{x_{1}=\ldots=x} .
$$

\footnotetext{
${ }^{2}$ The only restriction is that $D_{\mu}^{x_{i}} f$ makes sense.
} 


\subsection{Classical Correlator $\mathcal{O}\left(g_{\mathrm{YM}}^{0}\right)$}

In this section we evaluate the tree level correlator (3.3). For $n, m \neq 0$ it reads in terms of (3.5)

$\left\langle\mathcal{D}_{\mu \nu, n} \overline{\mathcal{D}}_{\rho \sigma, m}\right\rangle_{0}=\left.\frac{J^{-3}}{4 N_{0}^{2 J}} \sum_{i, j, r, s=1}^{J} q^{j-i} p^{r-s} \partial_{\mu}^{x_{i}} \partial_{\nu}^{x_{j}} \partial_{\rho}^{y_{r}} \partial_{\sigma}^{y_{s}}\left\langle\operatorname{tr} \prod_{k=1}^{J} Z\left(x_{k}\right) \operatorname{tr} \prod_{l=J}^{1} \bar{Z}\left(y_{l}\right)\right\rangle_{\text {tree }}\right|_{\substack{x_{1}=\ldots=x \\ y_{1}=\ldots=y}}$,

where $q=e^{2 \pi i n / J}$ and $p=e^{2 \pi i m / J}$. As before we understand the operator $\mathcal{D}_{\mu \nu, n}$ to be located at position $x$. The conjugated operator $\overline{\mathcal{D}}_{\rho \sigma, m}$ is implicitly understood to sit at point $y \neq x$ and their distance is denoted by $w=x-y$.

The evaluation of (3.9) goes as follows. At tree level the expectation value is given by $J$ propagators that connect the two traces. The first connection can be chosen arbitrarily but the others are determined by planarity. However, using (3.8), we can always relabel the coordinates in such a way that $Z\left(x_{1}\right)$ is connected to $\bar{Z}\left(y_{1}\right)$ and $Z\left(x_{2}\right)$ to $\bar{Z}\left(y_{2}\right)$ etc. This produces a factor of $J$ and another factor $N^{J}$ comes from the fact that the web of propagators consists of $J$ closed color lines. This leads to

$$
\left\langle\mathcal{D}_{\mu \nu, n} \overline{\mathcal{D}}_{\rho \sigma, m}\right\rangle_{0}=\left.\frac{J^{-2}}{4 N_{0}^{2 J}}\left(\frac{g_{\mathrm{YM}}^{2} N}{2}\right)^{J} \sum_{i j r s} q^{j-i} p^{r-s} \partial_{\mu}^{x_{i}} \partial_{\nu}^{x_{j}} \partial_{\rho}^{y_{r}} \partial_{\sigma}^{y_{s}} \prod_{k=1}^{J} I_{x_{k} y_{k}}\right|_{\substack{x_{1}=\ldots=x \\ y_{1}=\ldots=y}} .
$$

The function $I_{x y}$ is the propagator defined in the appendix in A.4a). Next we carry out the derivatives and set the coordinates equal. The resulting terms that have to be summed in the following are products of $J$ propagators where some have derivatives acting onto them. As an example let us pick

$$
I_{x y}^{J-3}\left(\partial_{\mu}^{x} \partial_{\rho}^{y} I_{x y}\right)\left(\partial_{\nu}^{x} I_{x y}\right)\left(\partial_{\sigma}^{y} I_{x y}\right)=\frac{8\left(4 w_{\mu} w_{\nu}-\delta_{\mu \nu} w^{2}\right) w_{\nu} w_{\sigma}}{(2 \pi)^{2 J}\left(w^{2}\right)^{J+4}}
$$

This structure occurs when $i$ and $r$ are equal and $i, j$, and $s$ are all different from each other. Accordingly the corresponding factor is

$$
\sum_{i, j, r, s=1}^{J} q^{j-i} p^{r-s} \delta_{i r}\left(1-\delta_{i j}\right)\left(1-\delta_{i s}\right)\left(1-\delta_{j s}\right)=-J^{2} \delta_{m n}+2 J \quad(n, m \neq 0)
$$

In the cases where the indices $n$ or $m$ are zero the evaluation of the correlator using (2.6) becomes even simpler since the derivatives can be performed after the summation. Up to 
terms of order $\mathcal{O}\left(J^{-1}\right)$ which are suppressed in the BMN limit, one finds $(n, m \neq 0)$

$$
\begin{aligned}
&\left\langle\mathcal{D}_{\mu \nu, n} \overline{\mathcal{D}}_{\rho \sigma, m}\right\rangle_{0}= \frac{1}{\left(w^{2}\right)^{J+2}}[ \\
&+\left(\delta_{n, m} J_{\mu \rho} J_{\nu \sigma}+\delta_{n,-m} J_{\mu \sigma} J_{\nu \rho}\right. \\
&\left.+\left(\delta_{\mu \nu}-\frac{2 w_{\mu} w_{\nu}}{w^{2}}\right)\left(\delta_{\rho \sigma}-\frac{2 w_{\rho} w_{\sigma}}{w^{2}}\right)\right], \\
&\left\langle\mathcal{D}_{\mu \nu, n} \overline{\mathcal{D}}_{\rho \sigma, 0}\right\rangle_{0}=-\frac{1}{\left(w^{2}\right)^{J+2}}\left(\delta_{\mu \nu}-\frac{2 w_{\mu} w_{\nu}}{w^{2}}\right) \frac{2 w_{\rho} w_{\sigma}}{w^{2}}, \\
&\left\langle\mathcal{D}_{\mu \nu, 0} \overline{\mathcal{D}}_{\rho \sigma, m}\right\rangle_{0}=-\frac{1}{\left(w^{2}\right)^{J+2}} \frac{2 w_{\mu} w_{\nu}}{w^{2}}\left(\delta_{\rho \sigma}-\frac{2 w_{\rho} w_{\sigma}}{w^{2}}\right), \\
&\left\langle\mathcal{D}_{\mu \nu, 0} \overline{\mathcal{D}}_{\rho \sigma, 0}\right\rangle_{0}=\frac{1}{\left(w^{2}\right)^{J+2}} \frac{4 w_{\mu} w_{\nu} w_{\rho} w_{\sigma}}{w^{4}},
\end{aligned}
$$

where the inversion matrix $J_{\mu \rho}(w)=\delta_{\mu \rho}-2 \frac{w_{\mu} w_{\rho}}{w^{2}}$ has emerged. But there are also other terms that spoil the general structure (2.8). Firstly, we have found an $n$-independent piece in the correlator of the non-zero modes and, secondly, there is an overlap between non-zero and zero modes. Both troubles can be cured by the following redefinition

$$
\mathcal{D}_{\mu \nu, n}^{\prime}:= \begin{cases}\mathcal{D}_{\mu \nu, n}-\mathcal{D}_{\mu \nu, 0}+\frac{1}{2} \delta_{\mu \nu} \mathcal{D}_{\kappa \kappa, 0} & \text { for } n \neq 0 \\ \mathcal{D}_{\mu \nu, 0} & \text { for } n=0\end{cases}
$$

which means in terms of the irreducible combinations (2.7)

$$
\begin{aligned}
\mathcal{D}_{(\mu \nu), n}^{\prime} & =\mathcal{D}_{(\mu \nu), n}-\mathcal{D}_{\mu \nu, 0}, \\
\mathcal{D}_{[\mu \nu], n}^{\prime} & =\mathcal{D}_{[\mu \nu], n}, \\
\mathcal{D}_{n}^{\prime} & =\mathcal{D}_{n}+\mathcal{D}_{\kappa \kappa, 0} .
\end{aligned}
$$

For these new operators we find in the BMN limit:

$$
\begin{aligned}
\left\langle\mathcal{D}_{\mu \nu, n}^{\prime} \overline{\mathcal{D}}_{\rho \sigma, m}^{\prime}\right\rangle_{0} & =\frac{\delta_{n, m} J_{\mu \rho} J_{\nu \sigma}+\delta_{n,-m} J_{\mu \sigma} J_{\nu \rho}}{\left(w^{2}\right)^{J+2}}, \\
\left\langle\mathcal{D}_{\mu \nu, n}^{\prime} \overline{\mathcal{D}}_{\rho \sigma, 0}^{\prime}\right\rangle_{0} & =\left\langle\mathcal{D}_{\mu \nu, 0}^{\prime} \overline{\mathcal{D}}_{\rho \sigma, m}^{\prime}\right\rangle_{0}=0, \\
\left\langle\mathcal{D}_{\mu \nu, 0}^{\prime} \overline{\mathcal{D}}_{\rho \sigma, 0}^{\prime}\right\rangle_{0} & =\frac{4 w_{\mu} w_{\nu} w_{\rho} w_{\sigma} / w^{4}}{\left(w^{2}\right)^{J+2}} .
\end{aligned}
$$

Now the index structure of $3.18 \mathrm{a}$ ) is exactly what conformal symmetry demands from primary operators. The particular structure of (3.18d) is not especially meaningful; it is just that term of the fourth derivative of $\langle Z \bar{Z}\rangle$ that is dominant for large $J$. The only important fact here about zero mode operators is, that there is no overlap between non-zero and zero modes $3.18 \mathrm{~b}$ ).

\subsection{Quantum Corrections $\mathcal{O}\left(g_{\mathrm{YM}}^{2}\right)$}

Now we turn to the evaluation of the quantum corrections to the $\mathcal{D} \overline{\mathcal{D}}$-correlators $(3.18$ ), which are summarized in (3.4). To begin with we investigate only the case $n, m \neq 0$; the 
other cases are treated in the summary on page 21. Explicitly we can write (3.4) as

$$
\left\langle\mathcal{D}_{\mu \nu, n} \overline{\mathcal{D}}_{\rho \sigma, m}\right\rangle_{1}=\left.\frac{J^{-3}}{4 N_{0}^{2 J}} \sum_{i j r s} q^{j-i} p^{r-s}\left\langle D_{\mu}^{x_{i}} D_{\nu}^{x_{j}} D_{\rho}^{y_{r}} D_{\sigma}^{y_{s}} \operatorname{tr} \prod_{k=1}^{J} Z\left(x_{k}\right) \operatorname{tr} \prod_{l=J}^{1} \bar{Z}\left(y_{l}\right)\right\rangle\right|_{\begin{array}{c}
x_{1}=\ldots=x \\
y_{1}=\ldots=y
\end{array}}
$$

if we consider out of the 16 terms contained in $D_{\mu}^{x_{i}} D_{\nu}^{x_{j}} D_{\rho}^{y_{r}} D_{\sigma}^{y_{s}}$ only the following ones:

$$
\begin{aligned}
& \partial_{\mu}^{x_{i}} \partial_{\nu}^{x_{j}} \partial_{\rho}^{y_{r}} \partial_{\sigma}^{y_{s}} \\
& \longrightarrow \quad\left\langle d_{\mu \nu, n}^{(0)} \bar{d}_{\rho \sigma, m}^{(0)}\right\rangle_{g_{\mathrm{YM}}^{2}} \\
& -i \partial_{\nu}^{x_{j}} \partial_{\rho}^{y_{r}} \partial_{\sigma}^{y_{s}}\left[A_{\mu},\right]^{x_{i}}-i \partial_{\mu}^{x_{i}} \partial_{\rho}^{y_{r}} \partial_{\sigma}^{y_{s}}\left[A_{\nu},\right]^{x_{j}} \\
& \longrightarrow \quad\left\langle d_{\mu \nu, n}^{(1)} \bar{d}_{\rho \sigma, m}^{(0)}\right\rangle_{g_{\mathrm{YM}}} \\
& -i \partial_{\mu}^{x_{i}} \partial_{\nu}^{x_{j}} \partial_{\sigma}^{y_{s}}\left[A_{\rho},\right]^{y_{r}}-i \partial_{\mu}^{x_{i}} \partial_{\nu}^{x_{j}} \partial_{\rho}^{y_{r}}\left[A_{\sigma},\right]^{y_{s}} \\
& \longrightarrow \quad\left\langle d_{\mu \nu, n}^{(0)} \bar{d}_{\rho \sigma, m}^{(1)}\right\rangle_{g_{\mathrm{YM}}} \\
& +\partial_{\nu}^{x_{j}} \partial_{\sigma}^{y_{s}}\left[A_{\mu},\right]^{x_{i}}\left[A_{\rho},\right]^{y_{r}}+\partial_{\nu}^{x_{j}} \partial_{\rho}^{y_{r}}\left[A_{\mu},\right]^{x_{i}}\left[A_{\sigma},\right]^{y_{s}} \\
& +\partial_{\mu}^{x_{i}} \partial_{\sigma}^{y_{s}}\left[A_{\nu},\right]^{x_{j}}\left[A_{\rho},\right]^{y_{r}}+\partial_{\mu}^{x_{i}} \partial_{\rho}^{y_{r}}\left[A_{\nu},\right]^{x_{j}}\left[A_{\sigma},\right]^{y_{s}} \\
& \left\langle d_{\mu \nu, n}^{(1)} \bar{d}_{\rho \sigma, m}^{(1)}\right\rangle_{\text {tree }} .
\end{aligned}
$$

Recall the discussion concerning the placement of the gauge field $A$, cf. (3.6), and the normal ordering (3.7). We begin with the first term in (3.20):

$$
\begin{aligned}
\left\langle d_{\mu \nu, n}^{(0)} \bar{d}_{\rho \sigma, m}^{(0)}\right\rangle_{g_{\mathrm{YM}}^{2}}= & \frac{J^{-3}}{4 N_{0}^{2 J}} \sum_{i j r s} q^{j-i} p^{r-s} \partial_{\mu}^{x_{i}} \partial_{\nu}^{x_{j}} \partial_{\rho}^{y_{r}} \partial_{\sigma}^{y_{s}} \\
& \left.\left\langle\operatorname{tr} \prod_{k=1}^{J} Z\left(x_{k}\right) \operatorname{tr} \prod_{l=J}^{1} \bar{Z}\left(y_{l}\right)\right\rangle_{g_{\mathrm{YM}}^{2}}\right|_{\substack{x_{1}=\ldots=x \\
y=\ldots=y}} .
\end{aligned}
$$

It is similar to the tree level expression, except that we now have to evaluate the expectation value with the appropriate interactions. The required vertices are conveniently written in terms of some functions (A.4) defined in the appendix. The scalar self-energy can be viewed as an interaction between two scalar fields [20]

$$
\begin{aligned}
\oint & \equiv\left\langle Z^{a}\left(x_{1}\right) \bar{Z}^{b}\left(x_{2}\right)\right\rangle_{\text {scalar self-energy }} \\
& =-2\left(\frac{g_{\mathrm{YM}}^{2}}{2}\right)^{2}\left(N \operatorname{tr} T^{a} T^{b}-\operatorname{tr} T^{a} \operatorname{tr} T^{b}\right)\left(Y_{112}+Y_{122}\right) .
\end{aligned}
$$

Additionally there are two interactions among four scalar fields, which are given by the 4 -scalar vertex

$$
\begin{aligned}
& \equiv\left\langle Z^{a}\left(x_{1}\right) Z^{b}\left(x_{2}\right) \bar{Z}^{c}\left(x_{3}\right) \bar{Z}^{d}\left(x_{4}\right)\right\rangle_{4 \text { scalar vertex }} \\
& =-\left(\frac{g_{\mathrm{YM}}^{2}}{2}\right)^{3}\left(\operatorname{tr}\left[T^{a}, T^{c}\right]\left[T^{b}, T^{d}\right]+\operatorname{tr}\left[T^{a}, T^{d}\right]\left[T^{b}, T^{c}\right]\right) X_{1234}
\end{aligned}
$$

and the gluon exchange vertex

$$
\begin{aligned}
\ldots & \equiv\left\langle Z^{a}\left(x_{1}\right) Z^{b}\left(x_{2}\right) \bar{Z}^{c}\left(x_{3}\right) \bar{Z}^{d}\left(x_{4}\right)\right\rangle_{\text {gluon exchange }} \\
& =-\left(\frac{g_{\mathrm{YM}}^{2}}{2}\right)^{3}\left(\operatorname{tr}\left[T^{a}, T^{c}\right]\left[T^{b}, T^{d}\right] H_{13,24}+\operatorname{tr}\left[T^{a}, T^{d}\right]\left[T^{b}, T^{c}\right] H_{14,23}\right) .
\end{aligned}
$$


Both expressions are again taken from [20]. These interactions are to be used to connect one $Z$ to one $\bar{Z}$ or two $Z$ 's to two $\bar{Z}$ 's in (3.21), respectively. The others are connected by ordinary propagators. Since we are only working at planar level, the four particle interactions have to occur between adjacent scalar fields. Using again the symmetry (3.8) we can move the vertices to our favorite position. We choose the four modules to connect $Z\left(x_{1}\right), Z\left(x_{2}\right), \bar{Z}\left(y_{1}\right)$, and $\bar{Z}\left(y_{2}\right)$. And it is convenient to place half of the scalar self-energy between $Z\left(x_{1}\right)$ and $\bar{Z}\left(y_{1}\right)$, and the other half between $Z\left(x_{2}\right)$ and $\bar{Z}\left(y_{2}\right)$. Selecting only the planar contributions all three interactions can be summarized in an effective four point vertex

$$
V_{x_{1} y_{1} x_{2} y_{2}}:=X_{x_{1} y_{1} x_{2} y_{2}}+H_{x_{1} y_{1}, x_{2} y_{2}}-\left(Y_{x_{1} x_{1} y_{1}}+Y_{x_{1} y_{1} y_{1}}\right) I_{x_{2} y_{2}}-I_{x_{1} y_{1}}\left(Y_{x_{2} x_{2} y_{2}}+Y_{x_{2} y_{2} y_{2}}\right)
$$

or graphically

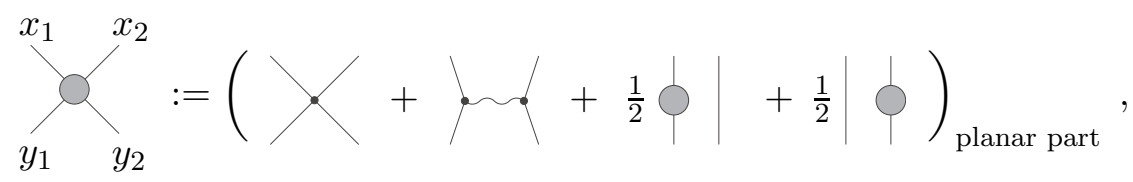

such that (3.21) becomes

$$
\begin{aligned}
\left\langle d_{\mu \nu, n}^{(0)} \bar{d}_{\rho \sigma, m}^{(0)}\right\rangle_{g_{\mathrm{YM}}^{2}}= & \frac{J^{-1}}{4 N_{0}^{2 J}}\left(\frac{g_{\mathrm{YM}}^{2} N}{2}\right)^{J+1} \\
& \times \sum_{i j r s} q^{j-i} p^{r-s} \partial_{\mu}^{x_{i}} \partial_{\nu}^{x_{j}} \partial_{\rho}^{y_{r}} \partial_{\sigma}^{y_{s}}\left[V_{x_{1} y_{1} x_{2} y_{2}} \prod_{k=3}^{J} I_{x_{k} y_{k}}\right]_{\begin{array}{c}
x_{1}=\ldots=x \\
y_{1}=\ldots=y
\end{array}} .
\end{aligned}
$$

The evaluation of this expression will simplify if we combine it with the other terms of (3.20). Therefore let us write $d_{\mu \nu, n}^{(1)}$ explicitly:

$$
\begin{aligned}
d_{\mu \nu, n}^{(1)}= & \left.\frac{J^{-3 / 2}}{2 N_{0}^{J}} \sum_{i j} q^{j-i} \partial_{\nu}^{x_{j}}\left[(-i) A_{\mu}\left(x_{i}\right),\right]^{x_{i}} \operatorname{tr} \prod_{k=1}^{J} Z\left(x_{k}\right)\right|_{\substack{x_{1}=\ldots=x \\
y_{1}=\ldots=y}} \\
& +\left(\mu \leftrightarrow \nu, q \rightarrow q^{-1}\right) .
\end{aligned}
$$

The application of the commutator to the trace produces $2 J$ terms. But by appropriate relabeling of the coordinates it is seen that all of them where $A_{\mu}\left(x_{i}\right)$ is inserted behind $Z\left(x_{i}\right)$ (for $\left.i=1, \ldots, J\right)$ are actually equal and all those where $A_{\mu}\left(x_{i}\right)$ is inserted in front of $Z\left(x_{i}\right)$ are equal as well. This enables us to write only two terms multiplied by a factor of $\mathrm{J}$

$$
\begin{aligned}
d_{\mu \nu, n}^{(1)}= & -\left.\frac{J^{-1 / 2}}{2 N_{0}^{J}} \sum_{j} q^{j} \partial_{\nu}^{x_{j}} \operatorname{tr} Z\left(x_{1}\right)\left[q^{-1}(-i) A_{\mu}\left(x_{1}\right)-q^{-2}(-i) A_{\mu}\left(x_{2}\right)\right] \prod_{k=2}^{J} Z\left(x_{k}\right)\right|_{x_{1}=\ldots=x} \\
& +\left(\mu \leftrightarrow \nu, q \rightarrow q^{-1}\right) .
\end{aligned}
$$

For the terms of the second line in (3.20) we compute the overlap with

$$
\bar{d}_{\rho \sigma, m}^{(0)}=\left.\frac{J^{-3 / 2}}{2 N_{0}^{J}} \sum_{r s} p^{r-s} \partial_{\rho}^{y_{r}} \partial_{\sigma}^{y_{s}} \operatorname{tr} \prod_{l=J}^{1} \bar{Z}\left(y_{l}\right)\right|_{y_{1}=\ldots=y} .
$$


The relevant interaction vertex is

$$
\begin{aligned}
& \equiv\left\langle(-i) A_{\mu}^{a}\left(x_{1}\right) Z^{b}\left(x_{2}\right) \bar{Z}^{c}\left(x_{3}\right)\right\rangle_{\text {gluon emission }} \\
& =\left(\frac{g_{\mathrm{YM}}^{2}}{2}\right)^{2} \operatorname{tr} T^{a}\left[T^{b}, T^{c}\right]\left(\partial_{\mu}^{2}-\partial_{\mu}^{3}\right) Y_{123}
\end{aligned}
$$

We insert it in such a way that the resulting diagrams are planar. This requires us to connect the gauge field $A$ to an adjacent $Z$, either to the left or to the right. The whole point is that these two possibilities can again be fitted nicely into an effective four point vertex

$$
W_{\mu, x_{1} y_{1} x_{2} y_{2}}:=I_{x_{1} y_{1}}\left(\partial_{\mu}^{x_{2}}-\partial_{\mu}^{y_{2}}\right) Y_{x_{1} x_{2} y_{2}}-I_{x_{2} y_{2}}\left(\frac{1}{2} \partial_{\mu}^{x_{1}}-\partial_{\mu}^{y_{1}}\right) Y_{x_{1} x_{1} y_{1}}
$$

or graphically

$$
\left.\sum_{y_{1}}^{x_{1}} \int_{y_{2}}^{x_{2}}:=(\mid\}|+|^{2} \cdot\right)_{\text {planar part }} .
$$

With this definition the overlap is written as

$$
\begin{aligned}
\left\langle d_{\mu \nu, n}^{(1)} \bar{d}_{\rho \sigma, m}^{(0)}\right\rangle_{g_{\mathrm{YM}}}= & \frac{J^{-1}}{4 N_{0}^{2 J}}\left(\frac{g_{\mathrm{YM}}^{2} N}{2}\right)^{J+1} \sum_{j r s} q^{j} p^{r-s} \partial_{\nu}^{x_{j}} \partial_{\rho}^{y_{r}} \partial_{\sigma}^{y_{s}} \\
& {\left[\left(q^{-1} W_{\mu, x_{1} y_{1} x_{2} y_{2}}+q^{-2} W_{\mu, x_{2} y_{2} x_{1} y_{1}}\right) \prod_{k=3}^{J} I_{x_{k} y_{k}}\right]_{\substack{x_{1}=\ldots=x \\
y_{1}=\ldots=y}} } \\
& +\left(\mu \leftrightarrow \nu, q \rightarrow q^{-1}\right)
\end{aligned}
$$

where a further factor of $J$ originated from singling out a particular $\bar{Z}$ the interaction takes place with. The third line in (3.20) is given by an analogous expression.

For the terms of the last two lines in $(3.20)$ we compute the overlap of 3.29 with itself, or more precisely with

$$
\begin{aligned}
\bar{d}_{\rho \sigma, m}^{(1)}= & -\left.\frac{J^{-1 / 2}}{2 N_{0}^{J}} \sum_{s} p^{-s} \partial_{\sigma}^{y_{s}} \operatorname{tr} \prod_{l=J}^{2} \bar{Z}\left(y_{l}\right)\left[p^{2}(-i) A_{\rho}\left(y_{2}\right)-p(-i) A_{\rho}\left(y_{1}\right)\right] \bar{Z}\left(y_{1}\right)\right|_{y_{1}=\ldots=y} \\
& +\left(\rho \leftrightarrow \sigma, p \rightarrow p^{-1}\right)
\end{aligned}
$$

Here we obtain the order $g_{\mathrm{YM}}^{2}$ diagrams already when all fields are connected by ordinary (tree level) propagators. Nevertheless we will think of the propagators connecting $Z\left(x_{1}\right)$ to $\bar{Z}\left(y_{1}\right), Z\left(x_{2}\right)$ to $\bar{Z}\left(y_{2}\right)$, and the $A$ 's to each other as a four point interaction in order to unify these terms most easily with the others from above. Similar to the true interactions (3.25) and (3.32), we therefore define the product of propagators as the following artificial 
four point vertex and introduce a corresponding graphical notation:

$$
\begin{aligned}
& \left.R_{\mu \rho, x_{1} y_{1} x_{2} y_{2}}:=\delta_{\mu \rho} I_{x_{1} y_{1}}^{2} I_{x_{2} y_{2}} \quad \triangleq \quad{\underset{\rho}{\mu}}_{y_{1}} y_{y_{2}}^{x_{1}}:=\right\} \text {, } \\
& \left.U_{\mu \rho, x_{1} y_{1} x_{2} y_{2}}:=-\delta_{\mu \rho} I_{x_{1} y_{1}} I_{x_{1} y_{2}} I_{x_{2} y_{2}} \stackrel{\wedge}{\mu}{\underset{y}{y_{1}} y_{2}}_{y_{2}}^{x_{1}}:=\mid\right\} \mid \text {. }
\end{aligned}
$$

Hence the correlator is given by

$$
\begin{aligned}
\left\langle d_{\mu \nu, n}^{(1)} \bar{d}_{\rho \sigma, m}^{(1)}\right\rangle_{\text {tree }}= & \frac{J^{-1}}{4 N_{0}^{2 J}}\left(\frac{g_{\mathrm{YM}}^{2} N}{2}\right)^{J+1} \sum_{j s} q^{j} p^{-s} \partial_{\nu}^{x_{j}} \partial_{\sigma}^{y_{s}} \\
& {\left[\left(q^{-1} p R_{\mu \rho, x_{1} y_{1} x_{2} y_{2}}+q^{-1} p^{2} U_{\mu \rho, x_{1} y_{1} x_{2} y_{2}}\right.\right.} \\
& \left.\left.\quad+q^{-2} p U_{\mu \rho, x_{2} y_{2} x_{1} y_{1}}+q^{-2} p^{2} R_{\mu \rho, x_{2} y_{2} x_{1} y_{1}}\right) \prod_{k=3}^{J} I_{x_{k} y_{k}}\right]_{\substack{x_{1}=\ldots=x \\
y_{1}=\ldots=y}} \\
& +\left(\mu \leftrightarrow \nu, q \rightarrow q^{-1}\right)+\left(\rho \leftrightarrow \sigma, p \rightarrow p^{-1}\right) \\
+ & \left(\mu \leftrightarrow \nu, \rho \leftrightarrow \sigma, q \rightarrow q^{-1}, p \rightarrow p^{-1}\right)
\end{aligned}
$$


Now we know all relevant terms in (3.19) and list them in their pictorial version

$$
\begin{aligned}
& \left\langle\mathcal{D}_{\mu \nu, n} \overline{\mathcal{D}}_{\rho \sigma, m}\right\rangle_{1}=\frac{J^{-1}}{4 N_{0}^{2 J}}\left(\frac{g_{\mathrm{YM}}^{2} N}{2}\right)^{J+1}\left\{\sum_{i j r s} q^{j-i} p^{r-s} \partial_{\mu}^{i} \partial_{\nu}^{j} \partial_{\rho}^{r} \partial_{\sigma}^{s}\left[\int_{y_{1}}^{x_{1}} \prod_{y_{2}}^{x_{2}} \prod_{k=3}^{J} I_{x_{k} y_{k}}\right]\right.
\end{aligned}
$$

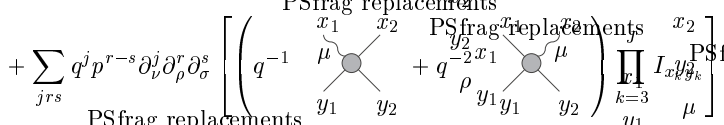

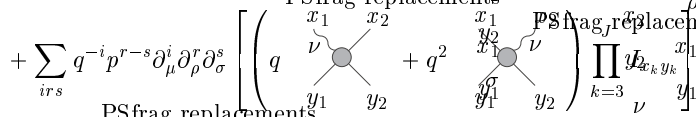

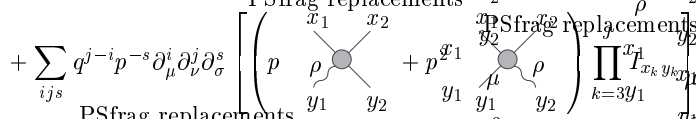

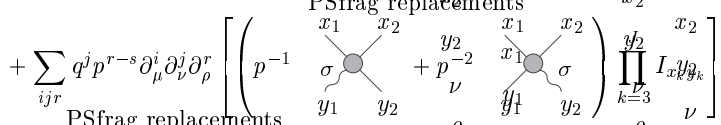

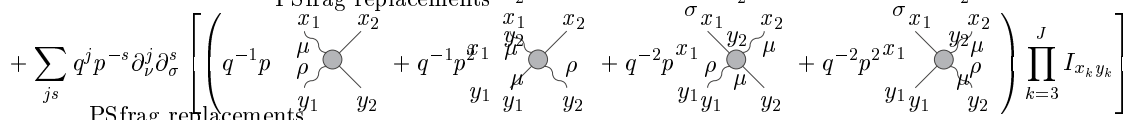

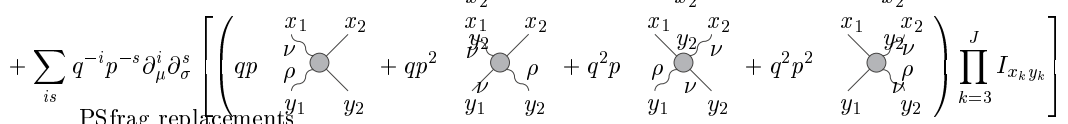

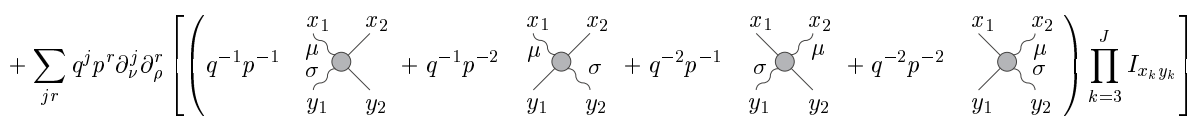

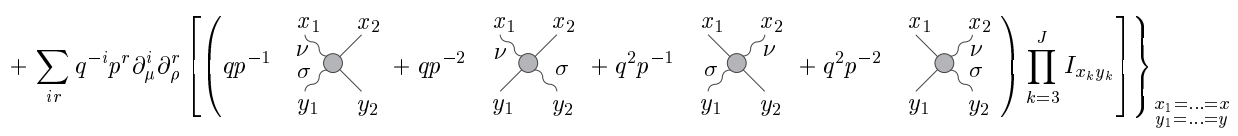

Although this looks rather complicated it is actually pretty intuitive. We derived the last eight lines of this expression be inserting gauge fields into the chain of $Z$ 's and moving them to the beginning of that chain. Afterwards the points $x_{1}, x_{2}, y_{1}$, and $y_{2}$ were connected by appropriate vertices and propagators. But effectively one may view these terms as a result of the application of some sort of operator to the legs of a four point vertex. One can further associate the same phase factors to this fictitious operator as for the derivatives that still have to be performed. This allows us to unify all lines of formula (3.39) into a single one by using the following graphical notation and definitions.

The fields $Z\left(x_{1}\right), Z\left(x_{2}\right), \bar{Z}\left(y_{1}\right)$, and $\bar{Z}\left(y_{2}\right)$ are connected by some four point vertex which we will simply denote by

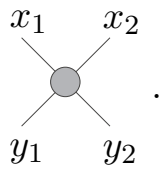

The operator we were talking about above does not receive a new symbol but assumes that of the covariant derivative and since in the end it has the same effect we will also refer to 
it as covariant derivative. Hence we write (3.39) concisely as

$$
\begin{aligned}
\left\langle\mathcal{D}_{\mu \nu, n} \overline{\mathcal{D}}_{\rho \sigma, m}\right\rangle_{1}= & \frac{J^{-1}}{4 N_{0}^{2 J}}\left(\frac{g_{\mathrm{YM}}^{2} N}{2}\right)^{J+1} \\
& \times\left.\sum_{i j r s} q^{j-i} p^{r-s} D_{\mu}^{x_{i}} D_{\nu}^{x_{j}} D_{\rho}^{y_{r}} D_{\sigma}^{y_{s}}\left[\sum_{y_{1}}^{x_{1}} \prod_{\substack{x_{2} \\
x_{2}}}^{J} I_{x_{k} y_{k}}\right]\right|_{\substack{x_{1}=\ldots=x \\
y_{1}=\ldots=y}}
\end{aligned}
$$

with the following meaning. The $D$ 's act onto the vertex as well as onto the propagators. Applied to the latter they are just ordinary partial derivatives. But when they are applied to the vertex a sum of terms is produced consisting of partial derivatives of the effective scalar vertex (3.25) and of the appropriately oriented effective vector vertices (3.32), (3.36), and (3.37). More precisely: The upper indices of the $D$ 's determine the effected legs of the vertex. On these legs $D$ can either act as partial derivatives or change the legs into a gluon line. One is supposed to write all possible combinations but to discard diagrams with more than one gluon line on one side (upper two or lower two legs). For example we have

$$
\begin{aligned}
D_{\mu}^{x_{2}} D_{\nu}^{x_{2}} D_{\rho}^{y_{1}} \sum_{y_{1}}^{x_{1}} & x_{y_{2}}^{x_{2}} \\
\equiv & \partial_{\mu}^{x_{2}} \partial_{\nu}^{x_{2}} \partial_{\rho}^{y_{1}} V_{x_{1} y_{1} x_{2} y_{2}}^{D_{\mu} D_{\nu}} \\
& +\partial_{\nu}^{x_{2}} \partial_{\rho}^{y_{1}} W_{\mu, x_{2} y_{2} x_{1} y_{1}}+\partial_{\mu}^{x_{2}} \partial_{\rho}^{y_{1}} W_{\nu, x_{2} y_{2} x_{1} y_{1}}+\partial_{\mu}^{x_{2}} \partial_{\nu}^{x_{2}} W_{\rho, y_{1} x_{1} y_{2} x_{2}} \\
& +\partial_{\nu}^{x_{2}} U_{\mu \rho, x_{2} y_{2} x_{1} y_{1}}+\partial_{\mu}^{x_{2}} U_{\nu \rho, x_{2} y_{2} x_{1} y_{1}} .
\end{aligned}
$$

After the derivatives have been applied all coordinates $x_{k}$ and $y_{l}$ are taken to coincide, respectively, which we depict by

$$
\oint_{\rho} \oint^{D_{\mu} D_{\nu}} .
$$

It is helpful to perceive that vertices with joint legs have certain symmetries under reflection. Clearly, all of these vertices are symmetric under reflection about the vertical axis. Under reflection about the horizontal axis the ones with an even number of derivatives are symmetric, whereas the ones with an odd number of derivatives are anti-symmetric. The quick argument for the latter statement is, that by Lorentz covariance they can only depend on an even or odd number of $w$ 's, respectively, and the reflection about the horizontal axis takes $w$ into $-w$. For instance, we have

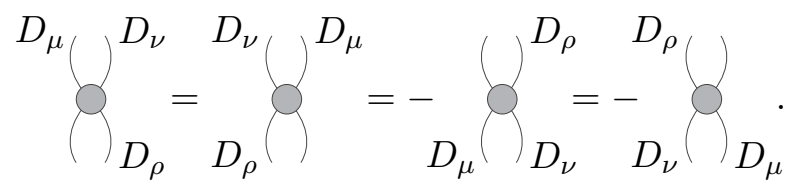


In the following we wish to show the evaluation of (3.41). We will successively consider the cases where none, one, two, three and all four derivatives act on the vertex. It will turn out that only the case with exactly two derivatives leads to non-vanishing contributions. If the number of derivatives is less than two, the vertices are zero and diagrams with more than two derivatives applied to the vertex do not contribute in the BMN limit.

\subsubsection{Vertex without any derivative}

The bare vertex is given by (3.25) which becomes

$$
V_{x y x y}=X_{x y x y}+H_{x y, x y}-4 Y_{x x y} I_{x y}
$$

by joining the legs, $x_{1}=x_{2}=x$ and $y_{1}=y_{2}=y$. But this combination vanishes identically which can be seen by utilizing the following relation between $X$ and $H[20]^{3}$ :

$$
\frac{H_{12,34}}{I_{12} I_{34}}=\frac{X_{1234}}{I_{13} I_{24}}-\frac{X_{1234}}{I_{14} I_{23}}+G_{1,34}-G_{2,34}+G_{3,12}-G_{4,12}
$$

with

$$
G_{1,34}=\frac{Y_{134}}{I_{14}}-\frac{Y_{134}}{I_{13}} .
$$

This relation simplifies in the limit $1,3 \rightarrow x$ and $2,4 \rightarrow y$. Taken into account that $Y_{x x y}$ and $X_{x y x y}$ contain logarithmic infinities whereas $1 / I_{x x}$ is quadratically zero it is easily shown that this implies

$$
=V_{x y x y}=0
$$

and therefore

$$
\left.\left\langle\mathcal{D}_{\mu \nu, n} \overline{\mathcal{D}}_{\rho \sigma, m}\right\rangle_{1}\right|_{\text {no derivative on vertex }}=0
$$

\subsubsection{Vertex with one derivative}

Without loss of generality let us investigate the case where the derivative sits at the upper left leg

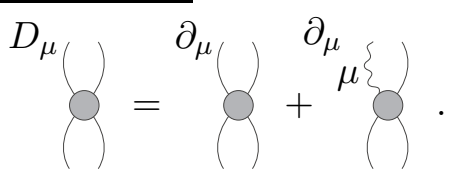

We show that both pieces are individually zero. For the first term this is quickly seen by an enjoyable calculation invoking our graphical notation. The vertex (3.48), where the legs are placed together, is only a function of $x$ (not $x_{1}$ and $x_{2}$ any more) and we may differentiate with respect to this coordinate. By Leibniz' rule this is related to the derivatives of the vertex before the legs were joint

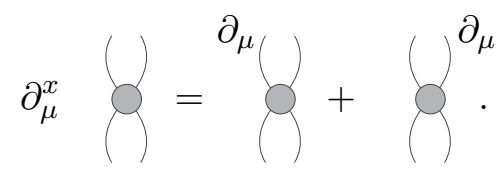

\footnotetext{
${ }^{3}$ This relation is written in eq. (A.7), however our function $H$ is defined to include the derivatives.
} 
Now, since (3.48) vanishes for all $x$, so does its derivative, i. e. (3.51) is zero. On the other hand both terms on the right hand side are equal by symmetry and therefore have to vanish as well

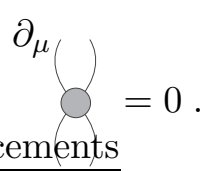

(The whole argument can of course also be rigorously proven by manipulating the corresponding analytic expressions.) The second vertex in (3.50) is immediately seen to vanish, if we set $x_{1}=x_{2}$ and $y_{1}=y_{2}$ in (3.32) and use $\partial_{\mu}^{x_{2}} Y_{x_{1} x_{2} y_{2}} \rightarrow \frac{1}{2} \partial_{\mu}^{x} Y_{x x y}$ :

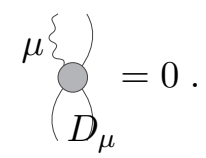

We conclude

$$
\left.\left\langle\mathcal{D}_{\mu \nu, n} \overline{\mathcal{D}}_{\rho \sigma, m}\right\rangle_{1}\right|_{\text {one derivative on vertex }}=0
$$

\subsubsection{Vertex with two derivatives}

This is the first case with non-zero vertices. As mentioned above it is the only case contributing to the anomalous dimension in the BMN limit. There are four diagrams with different placements of the two derivatives that we need to know:

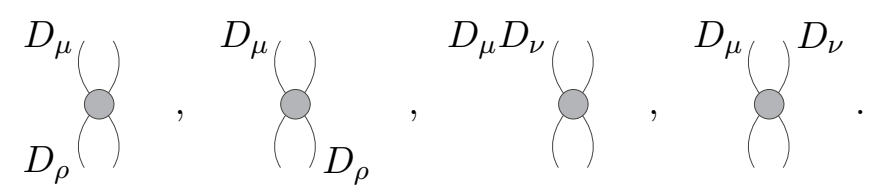

Let us sketch how the computation may be done. One resolves the meaning of the vertices in terms of derivatives of the scalar and vector vertices, (3.25), (3.32), (3.36), and (3.37). Then one uses their integral representations in terms of (A.4). It is advisable to change to momentum space, where one finds a generic two loop integral of the form

$$
\int \frac{d^{d} k}{(2 \pi)^{d}} \frac{d^{d} l}{(2 \pi)^{d}} \frac{\cdots}{k^{2} l^{2}(k-p)^{2}(l-p)^{2}(k-l)^{2}} .
$$

Here $d$ is the space-time dimension and $p$ the momentum of the Fourier transformation. The numerator is some function of $k, l$, and $p$ with two space-time indices. This kind of integral can conveniently be attacked with the aid of the mathematica package named TARCER [45]. Among many other things it is capable of reducing any two loop integral into a linear combination of known basic loop integrals which have unit numerator. For example we find for the first vertex in (3.55) the following momentum space representation

$$
\begin{aligned}
D_{\mu} & =\frac{p_{\mu} p_{\rho}}{p^{2}} \frac{3(d-4)(d-2)^{2} p^{2}\left[B^{(d)}(p)\right]^{2}-4\left(8 d^{3}-55 d^{2}+116 d-72\right) J^{(d)}(p)}{3(d-4)^{2}(d-1)} \\
& +\delta_{\mu \rho} \frac{-3(d-4) p^{2}\left[B^{(d)}(p)\right]^{2}+\left(3 d^{3}-13 d^{2}+14 d-16\right) J^{(d)}(p)}{3(d-4)^{2}(d-1)},
\end{aligned}
$$


where

$$
\begin{aligned}
B^{(d)}(p) & \equiv \int \frac{d^{d} k}{(2 \pi)^{d}} \frac{1}{k^{2}(k-p)^{2}}=\frac{1}{(4 \pi)^{d / 2}} \frac{\Gamma\left(2-\frac{d}{2}\right) \Gamma^{2}\left(\frac{d}{2}-1\right)}{\Gamma(d-2)} \frac{1}{\left(p^{2}\right)^{2-d / 2}}, \\
J^{(d)}(p) & \equiv \int \frac{d^{d} k}{(2 \pi)^{d}} \frac{d^{d} l}{(2 \pi)^{d}} \frac{1}{k^{2}(l-p)^{2}(k-l)^{2}}=\frac{1}{(4 \pi)^{d}} \frac{\Gamma^{3}\left(\frac{d}{2}-1\right) \Gamma(3-d)}{\Gamma\left(\frac{3 d}{2}-3\right)} \frac{1}{\left(p^{2}\right)^{3-d}} .
\end{aligned}
$$

We use the formula

$$
\int \frac{d^{d} p}{(2 \pi)^{d}} \frac{e^{i p w}}{\left(p^{2}\right)^{s}}=\frac{\Gamma\left(\frac{d}{2}-s\right)}{4^{s} \pi^{d / 2} \Gamma(s)} \frac{1}{\left(w^{2}\right)^{d / 2-s}}
$$

for the Fourier transformation and find

$$
\sum_{\rho}^{D_{\mu}}=2 I^{3}(w) J_{\mu \rho}(w) L_{\varepsilon}(w)
$$

with $L_{\varepsilon}(w):=-\frac{1}{\varepsilon}+\ln w^{-2}+1-\gamma_{E}-\ln \pi$ where $\gamma_{E}$ is Euler's constant. Eq. (3.61) has been written for $d=4-2 \varepsilon$ dimensions and all terms $\mathcal{O}(\varepsilon)$ have been neglected. The singular and constant (independent of $w$ ) terms disappear after renormalization of the operators. From (3.61) we can deduce the second vertex in (3.55) by a nice graphical reasoning. We differentiate (3.52) and (3.53) with respect to $y$

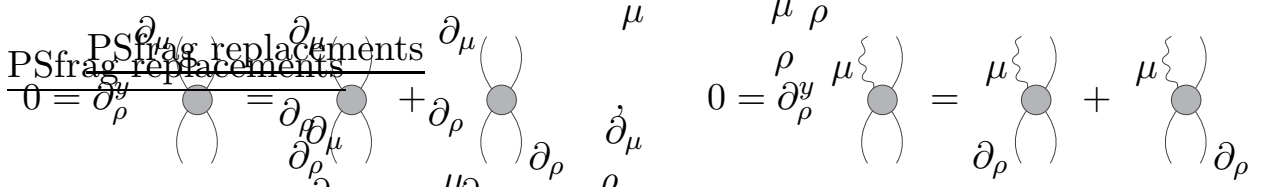

and also observe

$$
\mu\left\{\begin{array}{l}
\mu \\
\beta_{\rho}
\end{array}=-\delta_{\mu \rho} I_{x y}^{3}=-\begin{array}{l}
\mu\{ \\
\rho
\end{array}\right\} .
$$

This implies

$$
\begin{aligned}
& \beta_{D_{\rho}}^{D_{\mu}}=\oint_{\partial_{\rho}}^{\partial_{\mu}}+{ }^{\mu\{} \partial_{\partial_{\rho}}+\beta_{\rho}{ }^{\partial_{\mu}}{ }^{\mu} \beta_{\rho}
\end{aligned}
$$

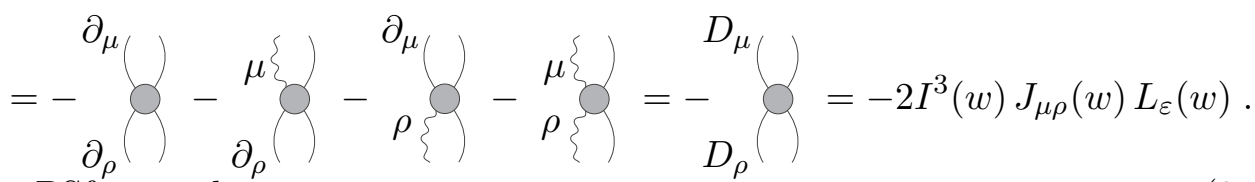

The third vertex in (3.55) has to be computed explicitly again. One finds an expression which is finite in four dimensions:

$$
D_{\mu} D_{\nu}=I^{3}(w) \delta_{\mu \nu} .
$$


The last vertex is the negative of the previous one which can be deduced by an argument similar to (3.64). In summary we list the vertices with two covariant derivatives

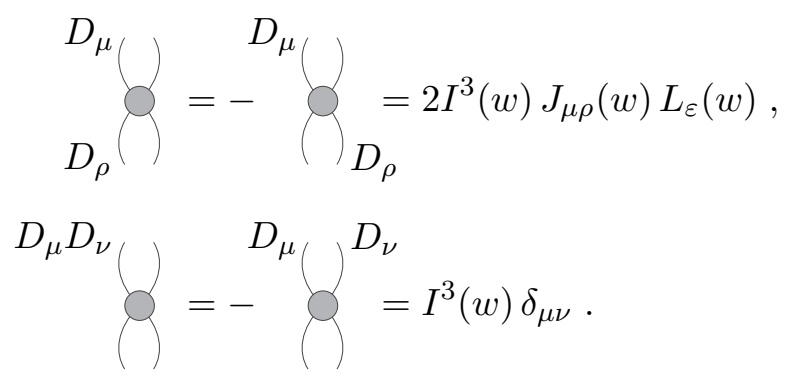

Now we can write down all terms in (3.41) which have two derivatives acting onto the vertex. The remaining two derivatives which have to be applied to the propagators are evaluated analogously to the tree level computation. The result is some lengthy function in $J$. But since we are only interested in the BMN limit where $J \rightarrow \infty$, we may expand this function in inverse powers of $J$. One finds that the first term in this expansion is of order $\mathcal{O}\left(1 / J^{2}\right)$. This is exactly what we were hoping to find because then the one-loop result possesses, compared to the tree level result, the additional factor of $\frac{N}{J^{2}}$, which is precisely the fixed parameter of the BMN limit (1.3), namely

$$
\begin{aligned}
\left.\left\langle\mathcal{D}_{\mu \nu, n} \overline{\mathcal{D}}_{\rho \sigma, m}\right\rangle_{1}\right|_{\substack{\text { two derivatives } \\
\text { on vertex }}} & \frac{g_{\mathrm{YM}}^{2} N n^{2}}{J^{2}} L_{\varepsilon}(w) \frac{\delta_{n, m} J_{\mu \rho} J_{\nu \sigma}+\delta_{n,-m} J_{\mu \sigma} J_{\nu \rho}}{\left(w^{2}\right)^{J+2}} \\
& -\frac{g_{\mathrm{YM}}^{2} N n^{2}}{4 J^{2}} \delta_{\mu \nu}\left(\delta_{\rho \sigma}-\frac{2 w_{\rho} w_{\sigma}}{w^{2}}\right) \\
& -\frac{g_{\mathrm{YM}}^{2} N m^{2}}{4 J^{2}} \delta_{\rho \sigma}\left(\delta_{\mu \nu}-\frac{2 w_{\mu} w_{\nu}}{w^{2}}\right)+N \mathcal{O}\left(J^{-3}\right) .
\end{aligned}
$$

The higher terms in this expansion disappear in the BMN limit, because they tend to zero faster than the single factor of $N$ in the numerator grows to infinity. Let us briefly discuss the origin of the pieces in (3.67). The two vertices in (3.66a or (3.66b) have the same space-time structure but receive different phase factors when inserted into (3.41), which for instance produces

$$
q^{-1}-q^{-2}=2 \pi i \frac{n}{J}+\mathcal{O}\left(J^{-2}\right), \quad p^{1}-p^{2}=-2 \pi i \frac{m}{J}+\mathcal{O}\left(J^{-2}\right) .
$$

As opposed to the scalar case where the impurities have to be connected among themselves, here it is not required that the two derivatives which are not involved in the vertex have to coincide at the same propagator. This allows terms which are off-diagonal in $n$ and $m$.

\subsubsection{Vertex with three derivatives}

There are the following vertices

$$
\left.D_{\rho} D_{\mu}, \quad \begin{array}{cc}
D_{\nu} & D_{\mu} D_{\nu} \\
& D_{\rho}
\end{array},{ }_{D_{\rho}}\right\}^{D_{\mu} D_{\nu}}
$$


from which all vertices with three derivatives can be obtained by appropriate reflections. We can avoid evaluating the vertices explicitly if we first expand the phase factors in $1 / J$. Explicitly we have

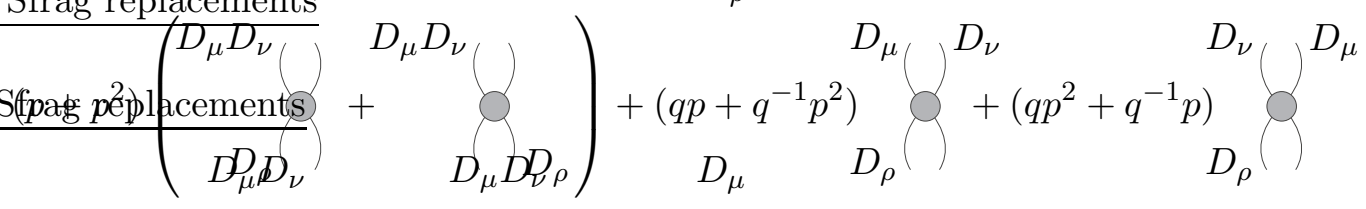

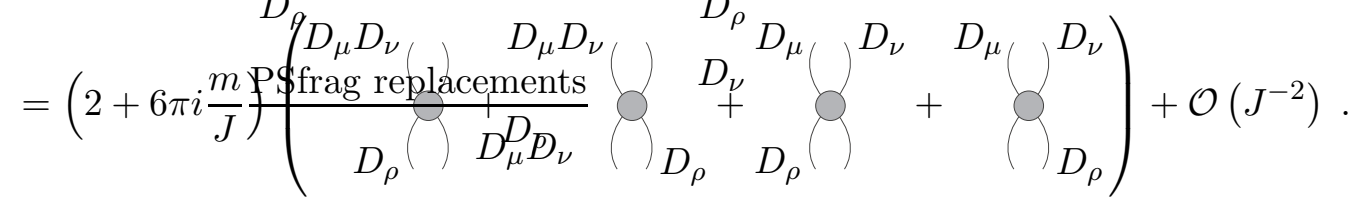

The higher terms may be disregarded in the BMN limit taken into account the additional factor $1 / J$ from the normalization and the fact that there do not emerge further powers of $J$ from the sum over the position of the fourth derivative. Thus we observe that only a particular sum of the vertices (3.69) could potentially contribute but one facilely shows that this is zero:

$$
\begin{aligned}
& \left.D_{\rho} Q_{\mu} D_{\nu}+\oint_{D_{\rho}}^{D_{\mu} D_{\nu}}+D_{\rho}\right\}^{D_{\mu}}+\oint_{D_{\rho}}^{D_{\nu}} \\
& =\partial_{\rho}^{y}(\underbrace{D_{\mu} D_{\nu}\left(Q^{D_{\mu}}+Q^{D_{\nu}}\right.}_{=0})+\partial_{\mu}^{x} \partial_{\nu}^{x}(\underbrace{\rho \xi}_{=0})
\end{aligned}
$$

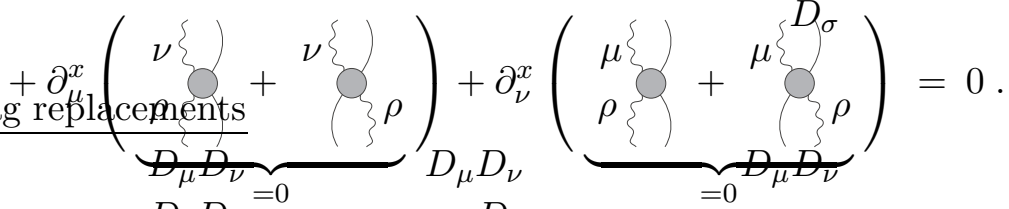

We have indicated which terms cancel each other by arguments given previously (cf. (3.66b), (3.53), and (3.63)). Hence we have

$$
\left.\left\langle\mathcal{D}_{\mu \nu, n} \overline{\mathcal{D}}_{\rho \sigma, m}\right\rangle_{1}\right|_{\text {three derivatives on vertex }}=N \mathcal{O}\left(J^{-3}\right) .
$$

\subsubsection{Vertex with four derivatives}

In this last case, there are four essentially different vertices

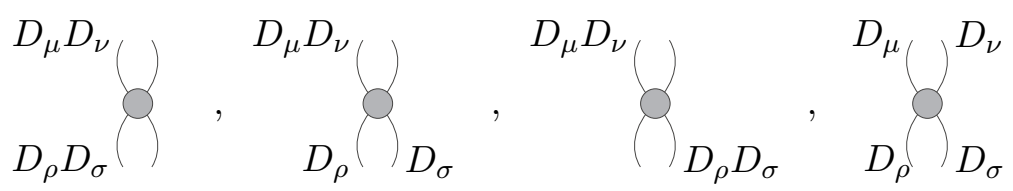

and their evaluation would be a rather tedious task. However, if we again expand the terms of (3.41) with four derivatives applied to the vertex into a power series in $1 / J$, we find that 
only the following combination of these vertices is important

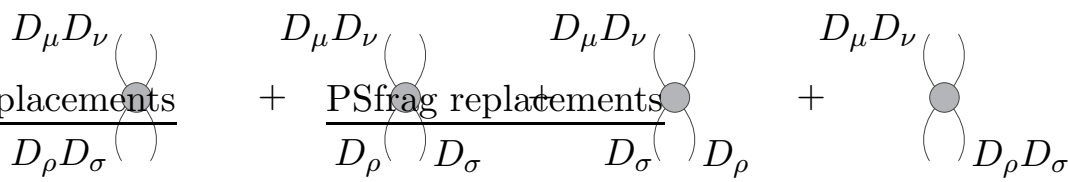

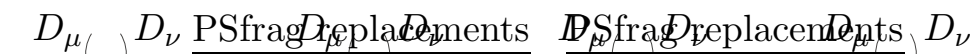

$$
\begin{aligned}
& { }_{D_{\rho} D_{\sigma}}^{+}+{ }_{D_{\rho}} \bigcap_{D_{\sigma}}+{ }_{D_{\sigma}} \bigcap_{D_{\rho}}+\bigcap_{D_{\rho} D_{\sigma}} \cdot
\end{aligned}
$$

In a couple of simple but paper consuming steps one converts this sum to

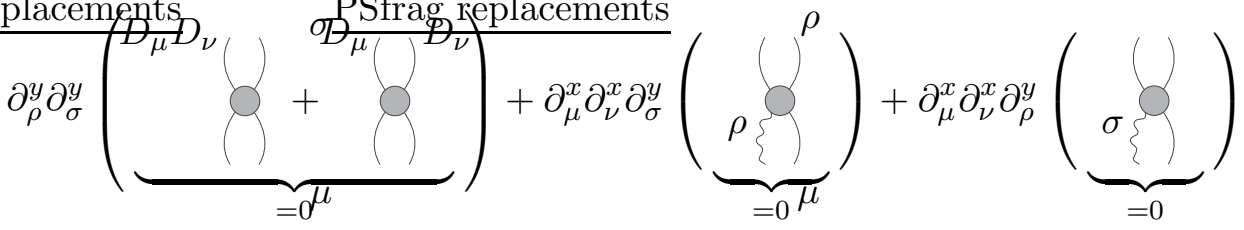

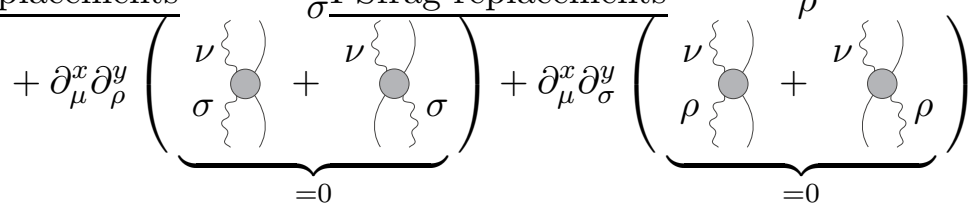

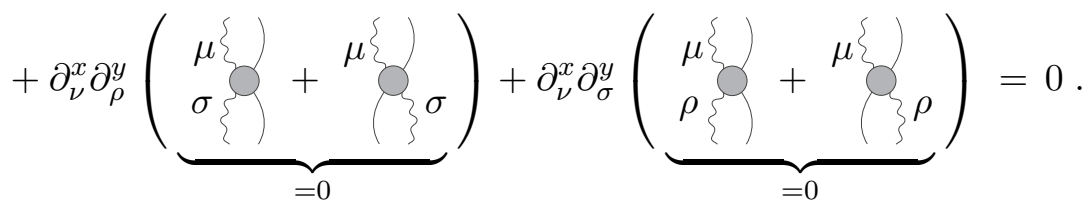

Thus we conclude that also

$$
\left.\left\langle\mathcal{D}_{\mu \nu, n} \overline{\mathcal{D}}_{\rho \sigma, m}\right\rangle_{1}\right|_{\text {four derivatives on vertex }}=N \mathcal{O}\left(J^{-3}\right) .
$$

\subsubsection{Summary}

For the non-zero mode operators $(n, m \neq 0)$ we have just found

$$
\begin{aligned}
\left\langle\mathcal{D}_{\mu \nu, n} \overline{\mathcal{D}}_{\rho \sigma, m}\right\rangle_{1}= & \lambda^{\prime} n^{2} L_{\varepsilon}(w) \frac{\delta_{n, m} J_{\mu \rho} J_{\nu \sigma}+\delta_{n,-m} J_{\mu \sigma} J_{\nu \rho}}{\left(w^{2}\right)^{J+2}} \\
& -\frac{\lambda^{\prime} n^{2}}{4} \delta_{\mu \nu}\left(\delta_{\rho \sigma}-\frac{2 w_{\rho} w_{\sigma}}{w^{2}}\right)-\frac{\lambda^{\prime} m^{2}}{4} \delta_{\rho \sigma}\left(\delta_{\mu \nu}-\frac{2 w_{\mu} w_{\nu}}{w^{2}}\right),
\end{aligned}
$$

where all higher terms of the $1 / J$ expansion have disappeared because the BMN limit was taken. We have combined the parameters according to (1.2).

Let us now come to the cases where $n=0$ or $m=0$ or both. Assume first $n \neq 0, m=0$ where the expression analogous to (3.41) reads

$$
\left\langle\mathcal{D}_{\mu \nu, n} \overline{\mathcal{D}}_{\rho \sigma, 0}\right\rangle_{1}=\left.\frac{J^{-2}}{4 N_{0}^{2 J}}\left(\frac{g_{\mathrm{YM}}^{2} N}{2}\right)^{J+1} \partial_{\rho}^{y} \partial_{\sigma}^{y} \sum_{i j} q^{j-i} D_{\mu}^{x_{i}} D_{\nu}^{x_{j}}\left[\sum_{y}^{x_{1}} \prod_{y=3}^{x_{2}} I_{x_{k} y}^{J}\right]\right|_{x_{1}=\ldots=x} .
$$


Since we may leave the derivatives with respect to $y$ to the very end, there are only terms with none, one or two (covariant) derivatives acting onto the upper two legs of the vertex. As a consequence the only contribution stems from (3.66b) and produces

$$
\left\langle\mathcal{D}_{\mu \nu, n} \overline{\mathcal{D}}_{\rho \sigma, 0}\right\rangle_{1}=\frac{\lambda^{\prime} n^{2}}{4} \delta_{\mu \nu} \frac{2 w_{\rho} w_{\sigma}}{w^{2}}
$$

If however $n=0$ and $m \neq 0$, the result is

$$
\left\langle\mathcal{D}_{\mu \nu, 0} \overline{\mathcal{D}}_{\rho \sigma, m}\right\rangle_{1}=\frac{\lambda^{\prime} m^{2}}{4} \delta_{\rho \sigma} \frac{2 w_{\mu} w_{\nu}}{w^{2}} .
$$

For $n=m=0$ there is no quantum correction at all since in this case we have a correlator of two (descendants of) protected operators. But this is also easy to comprehend from the computation since the vertex without derivatives has been shown to vanish:

$$
\left\langle\mathcal{D}_{\mu \nu, 0} \overline{\mathcal{D}}_{\rho \sigma, 0}\right\rangle_{1}=0
$$

Finally it remains to apply the transformation (3.14) in order to find the correlators of the redefined operators $(n, m \neq 0)$ :

$$
\begin{aligned}
\left\langle\mathcal{D}_{\mu \nu, n}^{\prime} \overline{\mathcal{D}}_{\rho \sigma, m}^{\prime}\right\rangle_{1} & =\lambda^{\prime} n^{2} L_{\varepsilon}(w) \frac{\delta_{n, m} J_{\mu \rho} J_{\nu \sigma}+\delta_{n,-m} J_{\mu \sigma} J_{\nu \rho}}{\left(w^{2}\right)^{J+2}}, \\
\left\langle\mathcal{D}_{\mu \nu, n}^{\prime} \overline{\mathcal{D}}_{\rho \sigma, 0}^{\prime}\right\rangle_{1} & =\frac{\lambda^{\prime} n^{2}}{4} \delta_{\mu \nu} \frac{2 w_{\rho} w_{\sigma}}{w^{2}}, \\
\left\langle\mathcal{D}_{\mu \nu, 0}^{\prime} \overline{\mathcal{D}}_{\rho \sigma, m}^{\prime}\right\rangle_{1} & =\frac{\lambda^{\prime} m^{2}}{4} \delta_{\rho \sigma} \frac{2 w_{\mu} w_{\nu}}{w^{2}}, \\
\left\langle\mathcal{D}_{\mu \nu, 0}^{\prime} \overline{\mathcal{D}}_{\rho \sigma, 0}^{\prime}\right\rangle_{1} & =0 .
\end{aligned}
$$

In this way we again got rid of the unwanted terms in (3.77). However, we could not abolish the overlap between non-zero and zero mode operators, which is basically due to the fact that (3.81) vanishes and cannot aid in the transformation (3.14). It is therefore necessary to perform another redefinition. This time it will involve the coupling constant $\lambda^{\prime}$, since the plan is to make use of the tree level correlator $(3.18 \mathrm{c})$ in order to remove the overlap at one-loop level. The suitable redefinition is $(n \neq 0)$

$$
\mathcal{D}_{\mu \nu, n}^{\prime \prime}:=\mathcal{D}_{\mu \nu, n}^{\prime}-\frac{1}{8} \lambda^{\prime} n^{2} \delta_{\mu \nu} \mathcal{D}_{\kappa \kappa, 0}^{\prime}=\mathcal{D}_{\mu \nu, n}-\mathcal{D}_{\mu \nu, 0}+\frac{1}{2} \delta_{\mu \nu}\left(1-\frac{\lambda^{\prime} n^{2}}{4}\right) \mathcal{D}_{\kappa \kappa, 0}
$$

and leads to

$$
\begin{aligned}
\left\langle\mathcal{D}_{\mu \nu, n}^{\prime \prime} \overline{\mathcal{D}}_{\rho \sigma, m}^{\prime \prime}\right\rangle_{1} & =\lambda^{\prime} n^{2} L_{\varepsilon}(w) \frac{\delta_{n, m} J_{\mu \rho} J_{\nu \sigma}+\delta_{n,-m} J_{\mu \sigma} J_{\nu \rho}}{\left(w^{2}\right)^{J+2}}, \\
\left\langle\mathcal{D}_{\mu \nu, n}^{\prime \prime} \overline{\mathcal{D}}_{\rho \sigma, 0}^{\prime \prime}\right\rangle_{1} & =\left\langle\mathcal{D}_{\mu \nu, 0}^{\prime \prime} \overline{\mathcal{D}}_{\rho \sigma, m}^{\prime \prime}\right\rangle_{1}=\left\langle\mathcal{D}_{\mu \nu, 0}^{\prime \prime} \overline{\mathcal{D}}_{\rho \sigma, 0}^{\prime \prime}\right\rangle_{1}=0 .
\end{aligned}
$$

The tree level correlators are not affected by this redefinition. 


\subsection{Anomalous dimension}

We add the quantum corrections (3.84) to the classical part of the correlators (3.18):

$$
\begin{aligned}
\left\langle\mathcal{D}_{\mu \nu, n}^{\prime \prime} \overline{\mathcal{D}}_{\rho \sigma, m}^{\prime \prime}\right\rangle & =\frac{\delta_{n, m} J_{\mu \rho} J_{\nu \sigma}+\delta_{n,-m} J_{\mu \sigma} J_{\nu \rho}}{\left(w^{2}\right)^{J+2}}\left(1+\lambda^{\prime} n^{2} L_{\varepsilon}(w)\right), \\
\left\langle\mathcal{D}_{\mu \nu, 0}^{\prime \prime} \overline{\mathcal{D}}_{\rho \sigma, 0}^{\prime \prime}\right\rangle & =\frac{4 w_{\mu} w_{\nu} w_{\rho} w_{\sigma} / w^{4}}{\left(w^{2}\right)^{J+2}}
\end{aligned}
$$

and without overlap between non-zero and zero modes. Recall that this result is only valid in the BMN limit and that it represents only the planar part of the correlator. In order to read off the anomalous dimension we actually have to renormalize the non-zero mode operators by

$$
\mathcal{D}_{\mu \nu, n}^{\mathrm{ren}}:=\mathcal{D}_{\mu \nu, n}^{\prime \prime}\left(1+\lambda^{\prime} f(\varepsilon)\right) \quad \text { with } \quad f(\varepsilon)=\frac{n^{2}}{2}\left(\frac{1}{\varepsilon}-1+\gamma_{E}+\ln \pi\right)
$$

This only amounts to the replacement $L_{\varepsilon}(w) \rightarrow \ln w^{-2}$. Thus, by comparison with (2.8), we find the anomalous dimension

$$
\delta \Delta_{n}=\lambda^{\prime} n^{2}
$$

for all $n \in \mathbb{Z}$. Note that this result is independent of the particular $\mathrm{SO}(4)$ irrep. Together with (2.3) we obtain

$$
\Delta_{n}-J=\Delta^{(0)}-J+\delta \Delta_{n}=2+\lambda^{\prime} n^{2}
$$

in accordance with (2.1).

\section{Conclusion}

Our aim was to find BMN operators that represent the plane-wave string states

$$
\left(\alpha_{n}^{\mu}\right)^{\dagger}\left(\alpha_{-n}^{\nu}\right)^{\dagger}\left|0, p^{+}\right\rangle
$$

where the indices $\mu, \nu$ belong to that $\mathrm{SO}(4)$ subgroup of the symmetries of the plane-wave background that used to be symmetries of the $A d S_{5}$ space before the BMN limit was taken. These states are generically modeled by operators with covariant derivative insertions [1]. In analogy to the scalar case we defined in (2.2) and (2.6) $(n \neq 0)$ :

$$
\begin{aligned}
& \mathcal{D}_{\mu \nu, n}:=\frac{J^{-1 / 2}}{2 N_{0}^{J}}\left[\sum_{p=1}^{J-1} \operatorname{tr}\left(D_{\mu} Z\right) Z^{p-1}\left(D_{\nu} Z\right) Z^{J-1-p} e^{2 \pi i n p / J}+\operatorname{tr}\left(D_{\mu} D_{\nu} Z\right) Z^{J-1}\right], \\
& \mathcal{D}_{\mu \nu, 0}:=\frac{J^{-5 / 2}}{2 N_{0}^{J}} \partial_{\mu} \partial_{\nu} \operatorname{tr} Z^{J} .
\end{aligned}
$$

While the zero mode operators are explicitly descendants of a primary operator, it turned out that the non-zero mode operators are not primary. We showed that they become 
primary operators by a slight modification (3.14):

$$
\begin{aligned}
\mathcal{D}_{\mu \nu, n}^{\prime}:=\frac{J^{-1 / 2}}{2 N_{0}^{J}}[ & \sum_{p=1}^{J-1} \operatorname{tr}\left(D_{\mu} Z\right) Z^{p-1}\left(D_{\nu} Z\right) Z^{J-1-p} e^{2 \pi i n p / J}+\operatorname{tr}\left(D_{\mu} D_{\nu} Z\right) Z^{J-1} \\
& \left.+\left(\frac{1}{2} \delta_{\mu \nu} \square-\partial_{\mu} \partial_{\nu}\right) \frac{1}{J^{2}} \operatorname{tr} Z^{J}\right] .
\end{aligned}
$$

These operators, (4.4) and (4.3), then had proper two point correlators from a conformal field theory point of view. However, at one-loop level the non-zero mode operators were not orthogonal to the zero mode operators. This required a second redefinition (3.83), which this time had to depend on the coupling constant $\lambda^{\prime}$. This might seem strange at first sight since this kind of redefinition is not required in the case of scalar impurities, where zero mode and non-zero mode operators do not overlap. This is because of the fact that the zero mode operator with scalar impurities is a primary operator whereas the one with derivative impurities is a descendent operator. But apart from that one should keep in mind that there is generally - also in the scalar case - a $\lambda^{\prime}$ dependent redefinition, namely when the operators are renormalized, cf. (3.87). Including all redefinitions we have found that (4.1) can be represented by

$$
\begin{aligned}
\mathcal{D}_{\mu \nu, n}^{\mathrm{ren}}:=\left(1+\lambda^{\prime} f(\varepsilon)\right) \frac{J^{-1 / 2}}{2 N_{0}^{J}}[ & \sum_{p=1}^{J-1} \operatorname{tr}\left(D_{\mu} Z\right) Z^{p-1}\left(D_{\nu} Z\right) Z^{J-1-p} e^{2 \pi i n p / J}+\operatorname{tr}\left(D_{\mu} D_{\nu} Z\right) Z^{J-1} \\
& \left.+\left(\frac{1}{2} \delta_{\mu \nu} \square-\partial_{\mu} \partial_{\nu}\right) \frac{1}{J^{2}} \operatorname{tr} Z^{J}-\frac{\lambda^{\prime} n^{2}}{8} \delta_{\mu \nu} \square \frac{1}{J^{2}} \operatorname{tr} Z^{J}\right], \\
\mathcal{D}_{\mu \nu, 0}:=\frac{J^{-5 / 2}}{2 N_{0}^{J}} \partial_{\mu} \partial_{\nu} \operatorname{tr} Z^{J} . & \text { (4.5a) }
\end{aligned}
$$

Their anomalous dimensions give exactly the masses of (4.1) at order $\mathcal{O}\left(\lambda^{\prime}\right)$.

Actually this kind of operators had been studied before. In [22] N. Beisert derived operators representing the state (4.1) by arguments using superconformal symmetry. They look different for different $\mathrm{SO}(4)$ irreps. The exponential phase factors are replaced by sine and cosine functions. These operators are well-defined also outside the BMN limit for finite $J$. However for the limit (1.3) where the BMN correspondence is supposed to hold, we have shown that (4.5) represents (4.1), at least up to one-loop order and in the planar limit.

A practical reason prefers (4.5) to the finite $\mathrm{J}$ BMN operators of [22]. If one wants to actually compute the anomalous dimensions from diagrams it proves advantageous to utilize the $q$-derivative of [19]. But then we need a phase factor $q$ with $q^{J}=1$ in order to retain the cyclicity of the trace and hence we are led to exponentials.

As we have shown at length in section 3 the form of the operators allowed us to write the two point correlation function in a way convenient for the one-loop calculation, cf. (3.41). The details are rather intricate but effectively it amounts to a correlator of $\operatorname{tr} Z^{J}$ 
and $\operatorname{tr} \bar{Z}^{J}$ with one generic vertex and four derivative operations acting onto the whole object. Working with this expression in the subsequent computations consisted in simple graphical manipulations following merely from Leibniz' rule and reflection symmetries. The results were the following. If none or one derivative act onto the vertex, the vertex vanishes. If three or all four derivatives act onto the vertex, the diagrams are negligible in the BMN limit. Only the case where two derivatives act onto the vertex contributes to the anomalous dimension.

Regrettably, eq. (3.41) could only be found so easily in the planar limit. Though one could find effective vertices in general, all the nice and valuable relations between them only hold in the planar case. Moreover, in our planar computation we could move all different interactions on top of each other (always occurring between $Z\left(x_{1}\right), Z\left(x_{2}\right), \bar{Z}\left(y_{1}\right)$ and $\left.\bar{Z}\left(y_{1}\right)\right)$ and see them frequently cancel each other. This will not be possible in general and one has a large number of effective vertices. And as opposed to the case of scalar impurities, this cancellation is believed not to take place before the summation over the impurity insertion points. This is basically due to an essential difference between derivative and scalar case, namely the fact that derivative impurities have non-vanishing overlap with the "background" field $Z$, whereas scalar impurities do not:

$$
\left\langle D_{\mu} Z(x) \bar{Z}(y)\right\rangle \neq 0 \quad ; \quad\left\langle\phi_{i}(x) \bar{Z}(y)\right\rangle=0 .
$$

It is left for the future to find the subset of all vertices that are in the end relevant for computations involving two derivative BMN operators. This will hopefully be the analogue of the effective scalar vertex such that the symmetry between the two cases would be more apparent.

\section{Acknowledgments}

My special thanks go to Jan Plefka and Niklas Beisert for introducing me to the topic, for valuable discussions and useful hints. For further helpful discussions I would like to thank Ari Pankiewicz, Matthias Staudacher, Charlotte Kristjansen and Markus Pössel.

\section{A. Appendix: Yang-Mills theory}

We use the following Euclidean action of $\mathcal{N}=4$ supersymmetric Yang-Mills theory in $d=4$ dimensions:

$$
\begin{aligned}
S=\frac{2}{g_{\mathrm{YM}}^{2}} \int d^{4} x \operatorname{tr}( & \frac{1}{4} F_{\mu \nu} F_{\mu \nu}+\frac{1}{2} D_{\mu} \phi_{I} D_{\mu} \phi_{I}-\frac{1}{4}\left[\phi_{I}, \phi_{J}\right]\left[\phi_{I}, \phi_{J}\right] \\
& \left.+\frac{1}{2} \bar{\psi} \Gamma_{\mu} D_{\mu} \psi-\frac{i}{2} \bar{\psi} \Gamma_{I}\left[\phi_{I}, \psi\right]\right)
\end{aligned}
$$

which we fix in Feynman gauge. The quantum fields are the gauge field $A_{\mu}^{a}(\mu=1, \ldots, 4)$, six scalars $\phi_{I}^{a}(I=1, \ldots, 6)$ and four Majorana spinors $\psi^{a}$. The field strength is given by $F_{\mu \nu}=\partial_{\mu} A_{\nu}-\partial_{\nu} A_{\mu}-i\left[A_{\mu}, A_{\nu}\right]$ and the covariant derivative by $D_{\mu}=\partial_{\mu}-i\left[A_{\mu}, \quad\right]$. 
All fields transform in the adjoint representation of the gauge group, which in our case is $\mathrm{U}(N)$. The index $a$ labels the generators $T^{a}$ of $\mathrm{U}(N)$ and assumes the values $0, \ldots, N^{2}-1$. The generators obey the Lie algebra

$$
\left[T^{a}, T^{b}\right]=i f^{a b c} T^{c}
$$

and are normalized to

$$
\operatorname{tr} T^{a} T^{b}=\delta^{a b}, \quad \sum_{a=0}^{N^{2}-1}\left(T^{a}\right)_{\alpha \beta}\left(T^{a}\right)_{\gamma \delta}=\delta_{\alpha \delta} \delta_{\beta \gamma} .
$$

In the BMN correspondence one singles out a $\mathrm{U}(1) \cong \mathrm{SO}(2)$ subgroup of the $\mathrm{SU}(4) \cong$ $\mathrm{SO}(6) \mathrm{R}$-symmetry group. Label the scalar fields such that this $\mathrm{U}(1)$ subgroup rotates $\phi_{5}$ and $\phi_{6}$ into each other. Then $Z:=\frac{1}{\sqrt{2}}\left(\phi_{5}+i \phi_{6}\right)$ carries unit charge with respect to this $\mathrm{U}(1)$. The complex conjugated field $\bar{Z}$ possesses charge -1 , whereas the remaining 4 scalar fields are neutral.

The propagators and vertices are conveniently written in terms of the following functions which were defined in [20]

$$
\begin{aligned}
I_{12} & :=\int \frac{d^{d} p}{(2 \pi)^{d}} \frac{e^{i p\left(x_{1}-x_{2}\right)}}{p^{2}}=\frac{1}{4 \pi^{d / 2}} \frac{\Gamma\left(\frac{d}{2}-1\right)}{\left[\left(x_{1}-x_{2}\right)^{2}\right]^{d / 2-1}} \stackrel{d \rightarrow 4}{\longrightarrow} \frac{1}{4 \pi^{2}} \frac{1}{\left(x_{1}-x_{2}\right)^{2}}, \\
Y_{123} & :=\int d^{d} u I_{1 u} I_{2 u} I_{3 u}, \\
X_{1234} & :=\int d^{d} u I_{1 u} I_{2 u} I_{3 u} I_{4 u}, \\
H_{12,34} & :=\left(\partial^{1}-\partial^{2}\right) \cdot\left(\partial^{3}-\partial^{4}\right) \int d^{d} u d^{d} v I_{1 u} I_{2 u} I_{u v} I_{v 3} I_{v 4} .
\end{aligned}
$$

The arguments have been written as indices with the meaning $1 \equiv x_{1}$ etc. The propagators for scalar fields and the gauge field are

$$
\begin{aligned}
& \left\langle Z^{a}(x) \bar{Z}^{b}(y)\right\rangle=\frac{g_{\mathrm{YM}}^{2}}{2} \operatorname{tr} T^{a} T^{b} I_{x y}, \\
& \left\langle A_{\mu}^{a}(x) A_{\nu}^{b}(y)\right\rangle=\frac{g_{\mathrm{YM}}^{2}}{2} \delta_{\mu \nu} \operatorname{tr} T^{a} T^{b} I_{x y},
\end{aligned}
$$

respectively. The required vertices are given in the main text.

\section{References}

[1] D. Berenstein, J. M. Maldacena and H. Nastase: Strings in flat space and pp waves from $N=$ 4 super Yang Mills, JHEP 0204 (2002) 013, hep-th/0202021

[2] O. Aharony, S. S. Gubser, J. M. Maldacena, H. Ooguri and Y. Oz: Large N field theories, string theory and gravity, Phys. Rept. 323 (2000) 183, hep-th/9905111

[3] R. Penrose: Any spacetime has a plane wave as a limit, Differential geometry and relativity, Reidel, Dordrecht, 1976, pp. 271-275 
[4] M. Blau, J. Figueroa-O'Farrill, C. Hull and G. Papadopoulos: Penrose limits and maximal supersymmetry, Class. Quant. Grav. 19 (2002) L87, hep-th/0201081

[5] G. 't Hooft: A Planar Diagram Theory For Strong Interactions, Nucl. Phys. B 72 (1974) 461

[6] C. Kristjansen, J. Plefka, G. W. Semenoff and M. Staudacher: A new double-scaling limit of $N=4$ super Yang-Mills theory and PP-wave strings, Nucl. Phys. B 643 (2002) 3, hep-th/0205033

[7] N. R. Constable, D. Z. Freedman, M. Headrick, S. Minwalla, L. Motl, A. Postnikov and W. Skiba: PP-wave string interactions from perturbative Yang-Mills theory, JHEP 0207 (2002) 017, hep-th/0205089

[8] D. J. Gross, A. Mikhailov and R. Roiban: A calculation of the plane wave string Hamiltonian from $N=4$ super-Yang-Mills theory, hep-th/0208231

[9] J. Pearson, M. Spradlin, D. Vaman, H. Verlinde and A. Volovich: Tracing the string: BMN correspondence at finite $J^{* * 2 / N} / N$, hep-th/0210102

[10] R. A. Janik: BMN operators and string field theory, Phys. Lett. B 549 (2002) 237, hep-th/0209263

[11] J. Gomis, S. Moriyama and J. Park: SYM description of SFT Hamiltonian in a pp-wave background, hep-th/0210153

[12] J. Gomis, S. Moriyama and J. Park: SYM Description of PP-wave String Interactions: Singlet Sector and Arbitrary Impurities, hep-th/0301250

[13] D. J. Gross, A. Mikhailov and R. Roiban: Operators with large $R$ charge in $N=4$ Yang-Mills theory, Annals Phys. 301 (2002) 31, hep-th/0205066

[14] G. Arutyunov and E. Sokatchev: Conformal fields in the pp-wave limit, JHEP 0208 (2002) 014, hep-th/0205270

[15] M. Bianchi, B. Eden, G. Rossi and Y. S. Stanev: On operator mixing in $N=4 S Y M$, Nucl. Phys. B 646 (2002) 69, hep-th/0205321

[16] C. S. Chu, V. V. Khoze and G. Travaglini: Three-point functions in $N=4$ Yang-Mills theory and pp-waves, JHEP 0206 (2002) 011, hep-th/0206005

[17] A. Santambrogio and D. Zanon: Exact anomalous dimensions of $N=4$ Yang-Mills operators with large $R$ charge, Phys. Lett. B 545 (2002) 425, hep-th/0206079

[18] M.-X. Huang: String interactions in pp-wave from $N=4$ super Yang Mills, Phys. Rev. D 66 (2002) 105002, hep-th/0206248

[19] U. Gürsoy: Vector operators in the BMN correspondence, hep-th/0208041

[20] N. Beisert, C. Kristjansen, J. Plefka, G. W. Semenoff and M. Staudacher: BMN correlators and operator mixing in $N=4$ super Yang-Mills theory, Nucl. Phys. B 650 (2003) 125, hep-th/0208178

[21] N. R. Constable, D. Z. Freedman, M. Headrick and S. Minwalla: Operator mixing and the BMN correspondence, JHEP 0210 (2002) 068, hep-th/0209002

[22] N. Beisert: BMN operators and Superconformal Symmetry, hep-th/0211032

[23] J. A. Minahan and K. Zarembo: The Bethe-ansatz for $N=4$ super Yang-Mills, hep-th/0212208 
[24] N. Beisert, C. Kristjansen, J. Plefka and M. Staudacher: BMN gauge theory as a quantum mechanical system, arXiv:hep-th/0212269

[25] R. R. Metsaev: Type IIB Green-Schwarz superstring in plane wave Ramond-Ramond background, Nucl. Phys. B 625 (2002) 70, hep-th/0112044

[26] R. R. Metsaev and A. A. Tseytlin: Exactly solvable model of superstring in plane wave Ramond-Ramond background, Phys. Rev. D 65 (2002) 126004, hep-th/0202109

[27] M. Spradlin and A. Volovich: Superstring interactions in a pp-wave background, Phys. Rev. D 66 (2002) 086004, hep-th/0204146

[28] R. Gopakumar: String interactions in PP-waves, hep-th/0205174

[29] Y. Kiem, Y. Kim, S. Lee and J. Park: pp-wave / Yang-Mills correspondence: An explicit check, Nucl. Phys. B 642 (2002) 389, hep-th/0205279

[30] P. Lee, S. Moriyama and J. Park: Cubic interactions in pp-wave light cone string field theory, Phys. Rev. D 66 (2002) 085021, hep-th/0206065

[31] M. Spradlin and A. Volovich: Superstring interactions in a pp-wave background. II, JHEP 0301 (2003) 036, hep-th/0206073

[32] C. S. Chu, V. V. Khoze and G. Travaglini: pp-wave string interactions from n-point correlators of BMN operators, JHEP 0209 (2002) 054, hep-th/0206167

[33] C. S. Chu, V. V. Khoze, M. Petrini, R. Russo and A. Tanzini: A note on string interaction on the pp-wave background, hep-th/0208148

[34] J. H. Schwarz: Comments on superstring interactions in a plane-wave background, JHEP 0209 (2002) 058, hep-th/0208179

[35] A. Pankiewicz: More comments on superstring interactions in the pp-wave background, JHEP 0209 (2002) 056, hep-th/0208209

[36] A. Pankiewicz and B. Stefanski: pp-wave light-cone superstring field theory, hep-th/0210246

[37] C. S. Chu, M. Petrini, R. Russo and A. Tanzini: String interactions and discrete symmetries of the pp-wave background, hep-th/0211188

[38] Y. H. He, J. H. Schwarz, M. Spradlin and A. Volovich: Explicit formulas for Neumann coefficients in the plane-wave geometry, hep-th/0211198

[39] R. Roiban, M. Spradlin and A. Volovich: On light-cone SFT contact terms in a plane wave, hep-th/0211220

[40] E. T. Akhmedov: On the relations between correlation functions in $S Y M / p p-$ wave correspondence, hep-th/0212297

[41] C. S. Chu and V. V. Khoze: Correspondence between the 3-point BMN correlators and the 3-string vertex on the pp-wave, hep-th/0301036

[42] H. Verlinde: Bits, matrices and 1/N, hep-th/0206059

[43] J. G. Zhou: pp-wave string interactions from string bit model, Phys. Rev. D 67 (2003) 026010, hep-th/0208232

[44] D. Vaman and H. Verlinde: Bit strings from $N=4$ gauge theory, hep-th/0209215

[45] R. Mertig and R. Scharf: TARCER: A mathematica program for the reduction of two-loop propagator integrals, Comput. Phys. Commun. 111 (1998) 265, hep-ph/9801383 